\title{
COMPLETELY PERIODIC DIRECTIONS AND ORBIT CLOSURES OF MANY PSEUDO-ANOSOV TEICHMUELLER DISCS IN Q(1,1,1,1)
}

\author{
PASCAL HUBERT, ERWAN LANNEAU, MARTIN MÖLLER
}

\begin{abstract}
Авstract. In this paper, we investigate the closure of a large class of Teichmüller discs in the stratum $Q(1,1,1,1)$ or equivalently, in a $\mathrm{GL}_{2}^{+}(\mathbb{R})$-invariant locus $\mathcal{L}$ of translation surfaces of genus three. We describe a systematic way to prove that the $\mathrm{GL}_{2}^{+}(\mathbb{R})$-orbit closure of a translation surface in $\mathcal{L}$ is the whole of $\mathcal{L}$. The strategy of the proof is an analysis of completely periodic directions on such a surface and an iterated application of Ratner's theorem to unipotent subgroups acting on an "adequate" splitting.

This analysis applies for example to all Teichmüller discs stabilized obtained by Thurston's construction with a trace field of degree three which moreover "obviously not Veech".

We produce an infinite series of such examples and show moreover that the favourable splitting situation does not arise everywhere on $\mathcal{L}$, contrary to the situation in genus two.

We also study completely periodic directions on translation surfaces in $\mathcal{L}$. For instance, we prove that completely periodic directions are dense on surfaces obtained by Thurston's construction.
\end{abstract}

\section{Contents}

Introduction

1. Background

2. Configurations

3. Complete periodicity

4. Detecting the closure: reduction steps

5. Surfaces arising from Thurston's construction

6. Examples

7. Surfaces with a $2 T_{\text {fix }} 2 \mathrm{C}$-direction and covering constructions

Date: October 26, 2018.

2000 Mathematics Subject Classification. Primary: 32G15. Secondary: 30F30, 57R30, 37D40.

Key words and phrases. Abelian differential, Veech group, Pseudo-Anosov diffeomorphism, Teichmüller disc, orbit closures. 


\section{INTRODUCTION}

For translation surfaces in genus two, $\mathrm{GL}_{2}^{+}(\mathbb{R})$-orbit closures, completely periodic translation surfaces and many more properties have been classified by Calta, McMullen and e.g. [EMS03], [HLe06]. For half-translation surfaces, i.e. for pairs $(X, q)$ of a Riemann surface and a quadratic differential, as well as for surfaces of genus $g \geq 3$ the situation is much more complicated. At present, all classification questions are open. In [HLM06] and in the present paper, we study translation surfaces belonging to $\mathcal{L}$, the hyperelliptic locus of the non hyperelliptic connected component of the stratum $\mathcal{H}(2,2)$ (the moduli space of Abelian differentials having two double zeroes). The locus $\mathcal{L}$ is closed and $\mathrm{GL}_{2}^{+}(\mathbb{R})$-invariant. It is more natural to study $\mathcal{L}$ than it sounds on a first reading since it is $\mathrm{GL}_{2}^{+}(\mathbb{R})$-equivariantly isomorphic to $Q(1,1,1,1)$, the principal stratum of quadratic differentials in genus 2. We are thus studying the next simplest cases besides translation surfaces in genus two.

Projections of $\mathrm{GL}_{2}^{+}(\mathbb{R})$-orbits of translation surfaces to Teichmüller space give rise to Teichmüller discs. The setwise stabilizer of a Teichmüller disc in the mapping class group is a subgroup of $\mathrm{SL}_{2}(\mathbb{R})$, that reflects the geometry of the original translation surface. See Section 1 for details on the basic notions and references.

We focus in this paper on Teichmüller discs that are stabilized by a pseudoAnosov diffeomorphism (pseudo-Anosov Teichmüller discs or pseudo-Anosov translation surfaces, for short). The Arnoux-Yoccoz example ([AY81], [Arn88]) is a pseudo-Anosov Teichmüller disc with many very exotic properties. In [HLM06], we proved that the $\mathrm{GL}_{2}^{+}(\mathbb{R})$-orbit closure of the Arnoux-Yoccoz Teichmüller disc is the whole locus $\mathcal{L}$. Rephrasing the previous result in the language of quadratic differentials, we proved the existence of a pseudo-Anosov Teichmüller disc with a dense $\mathrm{GL}_{2}^{+}(\mathbb{R})$-orbit in $Q(1,1,1,1)$. This is very different from the behavior described by McMullen for Abelian differentials in genus 2: every pseudo-Anosov disc is contained in the eigenform locus over a Hilbert modular surface.

In this paper, we describe a systematic way to prove that the $\mathrm{GL}_{2}^{+}(\mathbb{R})$-orbit closure of a surface in $\mathcal{L}$ is the whole locus. This analysis applies to a large family of pseudo-Anosov Teichmüller discs obtained by the Thurston (or Thurston-Veech) construction (see [Thu88, Vee89]). Recall that a direction is completely periodic on a translation surface if the surface is decomposed into a union of cylinders in this direction. We have

Theorem 0.1. Let $(X, \omega) \in \mathcal{L}$ be a surface given by Thurston's construction with trace field of degree 3. Let us assume that $(X, \omega)$ is not a Veech surface for the most obvious reason: there exists a completely periodic direction which is not parabolic. Then

$$
\overline{\mathrm{GL}_{2}^{+}(\mathbb{R}) \cdot(X, \omega)}=\mathcal{L}
$$


Without the trace field condition the statement is false. Counterexamples are given by translation surfaces that arise as coverings from genus one or genus two.

Of course, by the ergodicity of the geodesic flow on any stratum, the $\mathrm{GL}_{2}^{+}(\mathbb{R})$ orbit closure of a generic surface in $\mathcal{L}$ equals $\mathcal{L}$. While pseudo-Anosov Teichmüller discs as in the theorem above behave like generic surfaces, they have quite remarkable topological properties. Without restrictions on the trace field we show:

Theorem 0.2. Let $(X, \omega) \in \mathcal{L}$ be a surface stabilized by a pseudo-Anosov diffeomorphism. Let us assume that there exists a completely periodic direction. Then the set of completely periodic directions on $(X, \omega)$ is a dense subset of the circle $\mathbb{S}^{1}$.

Obviously, the conclusion fails for a generic surface in $\mathcal{L}$, see Proposition 3.4. Note that the last result applies to translation surfaces given by Thurston's construction. Combining these last two theorems, one gets:

Theorem 0.3. Let $(X, \omega) \in \mathcal{L}$ be a surface given by Thurston's construction with trace field of degree 3 . Then at least one of the following holds:

(1) The closure of the orbit $\mathrm{GL}_{2}^{+}(\mathbb{R}) \cdot(X, \omega)$ is the whole locus $\mathcal{L}$.

(2) The limit set of the Veech group $\operatorname{SL}(X, \omega)$ is the full circle.

Remark 0.3.1. We do not know if there exists a surface which satisfies these two properties at the same time.

Our results apply to an infinite family of surfaces, for instance a series of surfaces arising from Thurston's construction.

Reader's guide. We end this introduction by explaining the organization of the paper and by sketching a proof of the main results.

The strategy of the proof of Theorem 0.1 is to use an "adequate irrational" splitting on a surface $(Y, \eta)$ belonging to the closure of the $\mathrm{SL}_{2}(\mathbb{R})$-orbit of $(X, \omega)$. Such a splitting is given by four homologous saddle connections which decompose the surface into two fixed tori and two exchanged cylinders. We will call such a decomposition a $2 \mathrm{~T}_{\text {fix }} 2 \mathrm{C}$ splitting. Following the strategy presented in [Mc3] and [HLM06], we conclude by applying Ratner's theorem [Rat91] to a cyclic unipotent subgroup acting on the product of the space of pairs of area 1 lattices and the space of cylinders.

We use the non parabolic completely periodic direction to obtain the surface $(Y, \eta)$ by the following way. In Section 2 we describe all completely periodic configurations of surfaces in $\mathcal{L}$. For each of these configurations, in Section 4 , we apply Ratner's theorem to $\left(\mathrm{SL}_{2}(\mathbb{R}) / \mathrm{SL}_{2}(\mathbb{Z})\right)^{3}$ the space of triples of normalized lattices in order to get a resplitting of $(X, \omega)$ into $(Y, \eta)$ with an irrational $2 T_{f i x} 2 C$. This can only work under some mild irrationality hypothesis, which will be a 
consequence of the trace field condition and the existence of a non-parabolic direction.

We now give a sketch of the proof of Theorem 0.2. Following Calta-Smillie [CS07], the hypothesis implies that the Sah-Arnoux-Fathi (SAF)-invariant [Arn81] is zero for every direction of the holonomy field. Given a direction $\theta$ containing a cylinder fixed by the hyperelliptic involution, we remove this cylinder from the surface and obtain a genus 2 translation surface $Y$ with boundary. Using the fact that the SAF-invariant vanishes on $Y$ and the list of periodic configuration given in Section 2, we prove that the flow is periodic in the direction $\theta$ on $Y$.

In Section 6, we check the hypothesis of Theorem 0.1 for an infinite family of examples arising from Thurston's construction.

In the last section, we briefly discuss the existence of a $2 T_{\text {fix }} 2 \mathrm{C}$ splitting on surfaces belonging to $\mathcal{L}$.

Acknowledgments. We thank C. McMullen for comments on preliminary versions of this article and E. Nipper for a careful reading. This work was partially supported by the ANR Teichmüller "projet blanc" ANR-06-BLAN-0038.

\section{BACKGROUND}

In this section we review basic notions concerning translation surfaces, trace fields, J-invariant and SAF-invariant.

A translation surface (or flat surface) is a Riemann surface $X$ with finitely many singularities $P_{i}$, plus the choice of charts covering $X \backslash \cup\left\{P_{i}\right\}$, such that the transition functions are translations. Equivalently, a translation surface is given by a pair $(X, \omega)$ of a Riemann surface $X$ and a holomorphic one-form $\omega$. For half-translation surfaces the conditions are relaxed to demanding the transition functions to be $\pm \mathrm{id}$ composed with a translation. Equivalently, half-translation surfaces are given by a pair $(X, q)$ of a Riemann surface $X$ and a quadratic differential $q$.

A half-translation surface admits a ramified double covering, which is a translation surface and except for the introduction we will work exclusively with translation surfaces. Translation surfaces correspond bijectively to pairs $(X, \omega)$ of a Riemann surface $X$ and a holomorphic one-form. Similarly, half-translation surfaces correspond bijectively to pairs $(X, q)$, where $q$ is a quadratic differential. See e.g. [MT02] for a survey on these notions. The singularities correspond to the zeros of $\omega$ resp. of $q$ under this bijection.

There is a natural action of $\mathrm{GL}_{2}^{+}(\mathbb{R})$ on (half-) translation surfaces by postcomposing the integration charts with the corresponding linear map. This action respects the number and multiplicities of zeros, called the signature, of the onefrom (resp. the quadratic differential). Consequently the moduli space of pairs 
$(X, \omega)$ (respectively of pairs $(X, q)$ ), denoted by $\mathcal{H}$ or also by $\Omega M_{g}$ (respectively $\left.Q M_{g}\right)$ is stratified by the signature. The stratum $\mathcal{H}(2,2)$ has two connected components (see [KZ03]). One component is the hyperelliptic component. The locus $\mathcal{L}$ is a codimension 1 subspace of the non hyperelliptic component of the stratum $\mathcal{H}(2,2)$.

Straight lines in the translation charts are geodesics for the metric $|\omega|$. A maximal subset of $X$ swept out by parallel geodesics is called an (open) cylinder. Its closure will be bounded by a finite number of saddle connections, geodesics joining the singularities. We will say that a cylinder is simple if each of its boundaries consists of a unique saddle connection (joining possibly the two zeroes). A geodesic has a well defined direction in $\mathbb{C} \cong \mathbb{R}^{2}$ and the direction of a cylinder is the direction of any of its geodesics.

Consider all geodesics in a fixed direction $\theta$. This direction is called periodic if there is an open cylinder in this direction. $\theta$ is called completely periodic, if $X$ decomposes completely into cylinders and saddle connections in this direction. Note that there is also the notion ([Cal04] $)$ of a completely periodic surface $(X, \omega)$, a surface such that each direction that has a cylinder is automatically completely periodic. This notion will not be considered in the sequel of this paper.

A completely periodic direction is called parabolic, if the moduli of the cylinders in this direction are commensurable. Parabolic directions are important, since a composition of suitable powers of Dehn twists along the cylinders produces a diffeomorphism which is affine with respect to the charts given by $\omega$ ([Vee89]). We denote by $\operatorname{Aff}^{+}(X, \omega)$ the group of orientation-preserving affine diffeomorphisms and by $\operatorname{SL}(X, \omega)$ its image under the natural map to $\mathrm{SL}_{2}(\mathbb{R})$. This image is called affine group or Veech group of $(X, \omega)$. It is well-known, that a diffeomorphism in $\operatorname{Aff}^{+}(X, \omega)$ is pseudo-Anosov, if and only if its image in $\operatorname{SL}(X, \omega)$ is a hyperbolic element of $\mathrm{SL}_{2}(\mathbb{R})$. The trace field of $(X, \omega)$ is the field generated over $\mathbb{Q}$ by the traces of all elements in $\operatorname{SL}(X, \omega)$. One can also define the trace field by the following way. One defines the holonomy vectors to be the integrals of $\omega$ along the saddle connections. Let us denote by $\Lambda=\Lambda(\omega)$ the subgroup of $\mathbb{R}^{2}$ generated by holonomy vectors $\Lambda=\int_{H_{1}(X, \mathbb{Z})} \omega$. If $e_{1}, e_{2} \in \Lambda$ are two nonparallel vectors in $\mathbb{R}^{2}$, one defines the holonomy field $k$ to be the smallest subfield of $\mathbb{R}$ such that every element of $\Lambda$ may be written as $a e_{1}+b e_{2}$ with $a, b \in k$. It is known ([KS00] Theorem 28) that if $\operatorname{SL}(X, \omega)$ contains a psuedo-Anosov diffeomorphism, then the trace field of $(X, \omega)$ coincides with $k$. In particular, any direction $\theta$ of saddle connection belongs to the trace field.

If $P$ is a polygon in $\mathbb{R}^{2}$ with vertices $v_{1}, \ldots, v_{n}$ in counterclockwise order about the boundary of $P$, then the $J$-invariant of $P$ is $J(P)=\sum_{i=1}^{n} v_{i} \wedge v_{i+1}$ (with the dummy 
condition $\left.v_{n+1}=v_{1}\right)$. Here $\wedge$ is taken to mean $\wedge_{\mathbb{Q}}$ and $\mathbb{R}^{2}$ is viewed as a $\mathbb{Q}$-vector space. $J(P)$ is a translation invariant (e.g. $J(P+\vec{v})=J(P)$ ), thus this permits to define $J(X, \omega)$ by $\sum_{i=1}^{k} J\left(P_{i}\right)$ where $P_{1} \cup \cdots \cup P_{k}$ is a cellular decomposition of $(X, \omega)$ into planar polygons (see [KS00]).

We will also make use of the SAF-invariant of an interval exchange transformation $f$ as follows. We define a linear projection $J_{x x}: \mathbb{R}^{2} \wedge_{\mathbb{Q}} \mathbb{R}^{2} \rightarrow \mathbb{R} \wedge_{\mathbb{Q}} \mathbb{R}$ by

$$
J_{x x}\left(\left(\begin{array}{l}
a \\
b
\end{array}\right) \wedge\left(\begin{array}{l}
c \\
d
\end{array}\right)\right)=a \wedge c .
$$

If $f$ is an interval exchange transformation induced by the first return map of the vertical foliation on $(X, \omega)$ (on a transverse interval $I$ ) then let us define the SAFinvariant of $f$ by $S A F(f)=J_{x x}(X, \omega)$. Note that the definition does not depend of the choice of $I$ if the interval meets every vertical leaf (see [Arn81]). We will also say that $\operatorname{SAF}(f)$ is the SAF-invariant of $(X, \omega)$ in the vertical direction. We define in an obvious manner the SAF-invariant of $(X, \omega)$ in a direction of the trace field.

If $f$ is a periodic interval exchange transformation then $S A F(f)=0$. The converse is not true in general. But if $f$ is defined over 2 or 3 intervals and $S A F(f)=0$ then $f$ is periodic.

We now sketch the Thurston construction ([Thu88], see also [Vee89]) of surfaces] with pseudo-Anosov diffeomorphisms. Choose a pair $\left(\left\{\gamma_{i}\right\}_{i \in I},\left\{\gamma_{j}\right\}_{j \in J}\right)$ of multicurves, i.e. of sets of simple closed curves on $X$ such that $X \backslash\left(\cup_{i \in I} \gamma_{i} \cup \cup_{j \in f} \gamma_{j}\right)$ is a union of simply connected regions. Let $M_{r, s}=\left(\gamma_{r}, \gamma_{s}\right)$ for $r, s \in I \cup J$ be the symmetric intersection matrix. Choose integer weigts $m_{r}$ for $r \in I \cup J$ and let $\left(h_{r}\right)$ be the unique (by Perron-Frobenius) positive eigenvector satisfying

$$
\mu h_{r}=\sum_{s \in I \cup J} m_{r} M_{r s} h_{s}
$$

Now glue rectangles $\left[0, h_{r}\right] \times\left[0, h_{s}\right]$ according to the intersection pattern of the multicurves $\gamma_{i}, i \in I$, and $\gamma_{j}, j \in J$, to obtain a closed surface (with cone-type singularities arising from the corners).

Suitable powers of Dehn twist along the curves $\gamma_{i}, i \in I$ (resp. along the curves $\left.\gamma_{j}, j \in J\right)$ define two non-commuting parabolic elements in $\operatorname{SL}(X, \omega)$. Suitable products of them give hyperbolic elements in $\operatorname{SL}(X, \omega)$, thus the corresponding diffeomorphism is a pseudo-Anosov diffeomorphism. One of the main results of [HLa06] shows, that not all pseudo-Anosov diffeomorphisms arise in this way.

\footnotetext{
${ }^{1}$ Also known as bouillabaisse surfaces, after a talk by J.Hubbard, CIRM 2003
} 


\section{Configurations}

In this section, we classify (topologically) the configurations of completely periodic directions $\theta$ on a translation surface belonging to the hyperelliptic locus $\mathcal{L}$.

2.1. Statement of the result. Cut $X$ along the set of saddle connections in the direction $\theta$. The result is a set of cylinders. To reconstruct the surface $(X, \omega)$, we have to glue these cylinders according to a combinatorics which encode which part of a cylinder is glued to another part of a cylinder. We will call such a combinatorics by a configuration. For instance, in Figure 1 $1 \mathrm{a}$, the configuration presented has 3 cylinders (for the vertical direction $\theta=\pi / 2$ ). One cylinder is fixed by the involution (we have represented the Weierstrass points by small bullets) and the two others are exchanged. We label the intervals representing the same saddle connection on the surface by the same number. Horizontal saddle connections are identified by vertical translation. A simple computation, using the Euler characteristic, shows that the number of cylinders is bounded above by 4. In this section we will prove the following result.

Theorem 2.1. Let $(X, \omega)$ be a translation surface in the hyperelliptic locus $\mathcal{L}$. Let us assume that the vertical direction is completely periodic. Then all possible configurations of cylinders are prescribed by Figure 1. More precisely:

(1) There are four configurations with two cylinders:

- One configuration with two exchanged cylinders corresponding to Figure 1h.

- Three configurations with two fixed cylinders corresponding to Figure 1i, Figure 1j and Figure $1 \mathrm{k}$.

(2) There are four configurations with three cylinders:

- Two configurations with one fixed cylinder corresponding to Figure 1h and Figure 1b.

- Two configurations with three fixed cylinders corresponding to Figure 1k and Figure 1 d.

(3) There are three configurations with four cylinders:

- Two configurations with two simple cylinders corresponding to Figure1 and Figure 1f.

- One configuration with one simple cylinder corresponding to Figure 1g.

$\mathrm{n}$

Remark 2.1.1. If $(X, \omega)$ admits a one cylinder decomposition then Thurston's construction implies that either $\operatorname{SL}(X, \omega)$ is cyclic, generated by a parabolic element, or $\mathrm{SL}(X, \omega)$ is commensurable to $\mathrm{SL}_{2}(\mathbb{Z})$, e.g. $(X, \omega)$ is a covering of at orus ramified at over point (see Lemma 5.3).

We will use the following obvious lemma. 


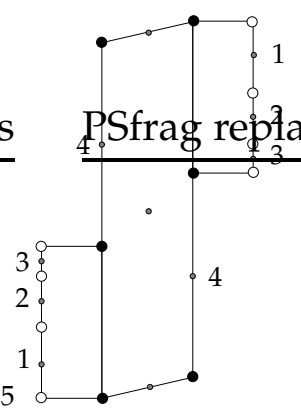

(a)

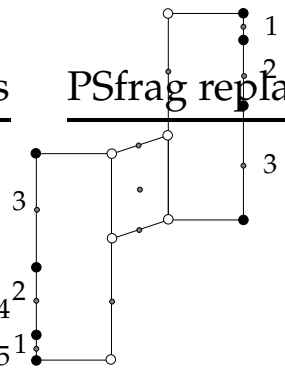

(b)

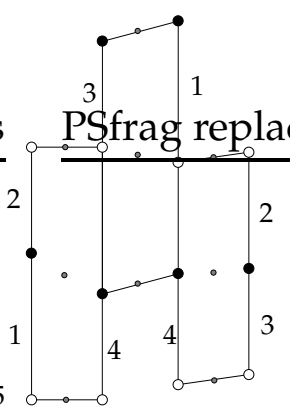

(c) $3 C_{\text {fix }}$

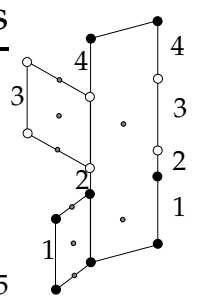

(d) $3 \mathrm{C}$

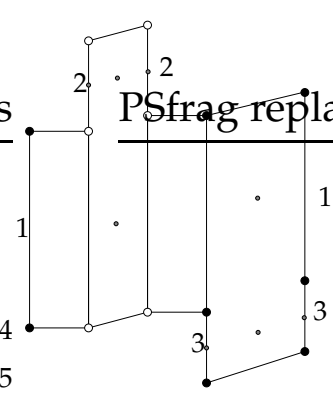

(e) $2 T_{\text {fix }} 2 \mathrm{C}$

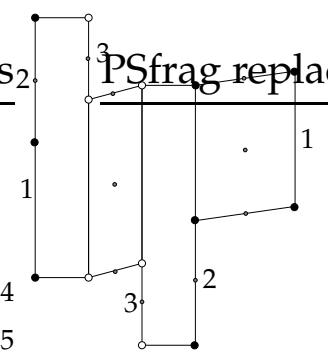

(f) $2 T 2 C_{\text {fix }}$

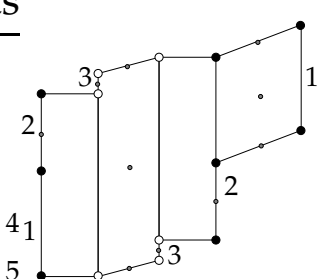

(g)

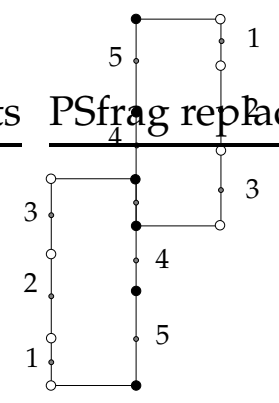

(h)

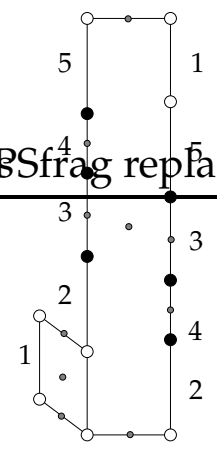

(i)

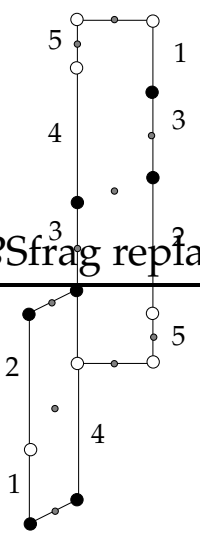

(j)

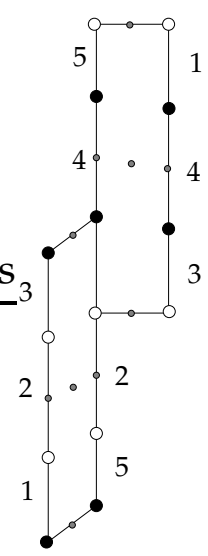

$(\mathrm{k})$

FIGURE 1. List of (topological) configurations of completely periodic surfaces in $\mathcal{L}$ (for the vertical direction).

Lemma 2.2. Let $(X, \omega) \in \mathcal{L}$ be a translation surface and let us assume that the vertical direction is completely periodic direction. Then:

(1) There exist 6 saddle connections joining a zero to another zero. 
(2) A Weierstrass point is located either on the middle of a saddle joining a zero to itself or on the core of a cylinder. In the last case there are exactly two Weierstrass points on the core curve.

(3) If no cylinder is fixed by the involution then there is no saddle connection connecting the two zeroes.

(4) If exactly one cylinder is fixed by the involution then there is at most one saddle connection connecting the two zeroes.

We will now give a proof of the main theorem of this section.

2.2. Proof of Theorem 2.1 in case of four cylinders. Let $(X, \omega)$ be a translation surface and let us assume that the vertical direction is completely periodic and decomposes the surface in four metric cylinders. Clearly the number of fixed cylinders (with respect to the hyperelliptic involution) is 0 or 2 . Indeed if all the cylinders are fixed then each cylinder will possess two fixed points (for the involution). Recall that the two zeroes are fixed, thus the hyperelliptic involution should possess $4 \times 2+2=10$ fixed points which is impossible.

Claim 2.3. There are exactly two fixed cylinders.

Proof of the Claim. Assume not. Then from above discussion, there is no fixed cylinder. Thus all the Weierstrass points (except for the two singularities) are located on the middle of the saddle connection. In particular, there is no saddle connection connecting the two zeroes.

Note that a Weierstrass point cannot be located on the boundary of a simple cylinder. Therefore there is no simple cylinder on such a configuration. Thus the only possible configuration is the following one: four cylinders, each of them having three saddle connections on its boundaries. It is then not hard to check that there is no possible configuration with 8 fixed points for the involution. The claim is proven.

Thus, a configuration with four cylinders must have exactly two fixed cylinders.

Claim 2.4. The number of simple cylinders is 1 or 2.

Proof of the Claim. Assume not. If there are four simple cylinders then obviously $(X, \omega)$ is a torus, which is impossible.

If there is no simple cylinder then the number of saddle connections is at least 8 for the two fixed cylinders and 6 for the other two. Finally the number of saddle connections is at least $8+6=14>12$, which is a also a contradiction.

Hence there is either 1 (necessarily fixed) simple cylinder, or 2 (fixed or exchanged) simple cylinders. These three possibilities lead to three configurations. 
It is not hard to show that the three possible cases are respectively given by Figure $1 \mathrm{~g}-1 \mathrm{f}-1 \mathrm{k}$. The last two configurations will be respectively called $2 \mathrm{~T} 2 \mathrm{C}_{\mathrm{fix}}$ and $2 T_{\text {fix }} 2 C$.

2.3. Proof of Theorem 2.1 in case of three cylinders. Let $(X, \omega)$ be a translation surface and let us assume that the vertical direction is completely periodic and decomposes the surface in three metric cylinders. Clearly the number of fixed cylinders (with respect to the hyperelliptic involution) is 1 or 3.

Claim 2.5. Let us assume that only one cylinder is fixed by the involution. Then the number of simple cylinders is 0 or 1 .

Proof of the Claim. Suppose not. Then the configuration for the vertical direction must contain two or three simple cylinders. In the last case $X$ is a torus, which is impossible.

If the configuration contains only two simple cylinders, they are necessarily exchanged. Thus each boundary of the fixed cylinder must possess exactly four saddle connections. Note that a Weierstrass point can not be located on the boundary of a simple cylinder. Let us count the number of Weierstrass points:

(1) the two zeroes.

(2) two Weierstrass points on the core curve of the fixed cylinder.

(3) at most two Weierstrass points on the middle of the saddle connections of the fixed cylinder.

Thus there are at most $6<8$ Weierstrass points which is a contradiction.

Hence for such a configuration, there is no simple cylinder or one fixed simple cylinder. It is then not difficult to check that theses two cases lead respectively to the configurations presented in Figure 1a and in Figure 1b.

Claim 2.6. Let us assume that the three cylinders are fixed by the involution. Then the number of simple cylinders is 0 or 2 .

Proof of the Claim. Assume not. Then the configuration contains one fixed simple cylinder. By a straightforward calculation, one proves that no possible configuration can occur in the hyperelliptic locus $\mathcal{L}$.

Thus in that case, the configuration possesses either three (non simple) fixed cylinders or two simples and one other (all fixed) cylinders. It is then easy to see that these two cases lead respectively to configurations presented in Figure $1 \mathrm{c}$ and in Figure 1d.

The proof of the theorem in case of two cylinders is left to the reader as an exercise. 


\section{Complete Periodicity}

We recall the following result by H. Masur (see [Mas86, MT02]):

Theorem 3.1 (Masur). Let $(X, \omega)$ be a translation surface. There is a dense set of periodic directions on $(X, \omega)$.

In this section, we prove the following strengthenings for translation surfaces in the locus $\mathcal{L}$ :

Theorem 3.2. Let $(X, \omega)$ be a translation surface that belongs to the hyperelliptic locus $\mathcal{L}$. There is a dense set of periodic directions $\theta \in \mathbb{S}^{1}$ such that, in the direction $\theta, \omega$ has a metric cylinder containing a Weierstrass point.

Theorem 3.3. Let $(X, \omega)$ be a translation surface in the hyperelliptic locus $\mathcal{L}$. Let us assume that the Teichmüller disc of $(X, \omega)$ is stabilized by a pseudo-Anosov diffeomorphism and that there exists a completely periodic direction. Then every periodic direction that contains a cylinder invariant by the hyperelliptic involution is completely periodic. Moreover the set of completely periodic directions is dense in $\mathbb{S}^{1}$.

Remark 3.3.1. Theorem 3.3 immediately implies Theorem 0.2 .

The statement of Theorem 3.3 is a weak form of the completely periodicity studied by Calta in genus 2 (see [Cal04]).

Nevertheless, it is not true that the hypothesis of the theorem implies that the surface is completely periodic in the sense of Calta. A direction with a splitting of the surface into two cylinders and two non periodic tori was exhibited on the Arnoux-Yoccoz example see [HLM06]. Similar constructions can be done for surfaces arising from Thurston's construction.

The following obvious remark shows that the behaviour stated in Theorem 3.3 is indeed special.

Proposition 3.4. Almost every surface in any stratum does not admit a completely periodic direction.

Proof. Complete periodicity can be expressed via proportionality of a non-empty set of relative periods. Since any stratum admits a coordinate system given by integration of a basis of relative periods, surfaces with a completely periodic direction are given by a linear subspace of positive codimension in this coordinate system. Since there are only countably many choices of such a basis, the set in question is of Lebesque measure zero.

\subsection{Proof of Theorem 3.2 .}

Definition 3.5. A slit is a geodesic segment embedded on a translation surface (in particular, it has no self-intersection and its end points differ). A slit torus is a flat torus with a marked point where a slit starting from the marked point is removed. 
The key ingredient in the proof is the following lemma.

Lemma 3.6. Let $(X, \omega)$ be a translation surface of area 1 in the hyperelliptic locus $\mathcal{L}$. Let us assume that the vertical direction is periodic, and, let $L$ be the length of the shortest vertical saddle connection. There is a direction $\theta$ with $|\cos (\theta)| \leq 1 / L^{2}$ such that, in the direction $\theta$, the surface $(X, \omega)$ has a metric cylinder containing a Weierstrass point.

Proof. If a vertical cylinder is invariant by the hyperelliptic involution, there is nothing to prove. Otherwise, the vertical direction contains two cylinders $C_{1}$ and $\mathrm{C}_{2}$ exchanged by the involution.

Section 2 describes the completely periodic vertical directions. In fact, it also gives all the types of periodic directions. By rescaling, we may suppose that the integral along the core curve of $C_{1}$ (and hence also of $C_{2}$ ) is in $\mathbb{Q} \cdot i$, while the width is in $\mathbb{Q}$. Hence there is a surface $(Y, \eta)$ arbitrarily close to $(X, \omega)$ with cylinders $C_{i}$ of the same size as those on $X$ and with relative periods in $\mathbb{Q}[i]$. The surface $(Y, \eta)$ is square-tiled by construction. Hence its vertical direction is completely periodic. A path between $(X, \omega)$ and $(Y, \eta)$ fixing $C_{1}$ and $C_{2}$ gives a way of describing $(X, \omega)$ as a deformation of a completely periodic surface, which leaves the cylinders $C_{1}$ and $C_{2}$ untouched.

The periodic configurations with two cylinders exchanged by the hyperelliptic involution are listed in Figure 1,

Using this list, we remark that $X \backslash\left(C_{1} \cup C_{2}\right)$ falls into the following list:

(1) a torus cut along a slit (Figure 1 1 a)

(2) a periodic cylinder (Figure $1 \mathrm{~b}$ )

(3) a union of two tori $T_{1}$ and $T_{2}$ cut along vertical slits (Figure 1e)

(4) a union of two periodic cylinders (Figure $1 \mathrm{f}$ )

(5) a slit torus and a periodic cylinder (Figure $1 \mathrm{~g}$ )

(6) empty (Figure 1h).

All the connected components of $X \backslash\left(C_{1} \cup C_{2}\right)$ are invariant under the hyperelliptic involution. Thus the vertical direction contains a periodic cylinder fixed by the involution when $X \backslash\left(C_{1} \cup C_{2}\right)$ is a cylinder (case (2)) or a union of a cylinder and a torus (case (5)).

Consequently, we assume that $X \backslash\left(C_{1} \cup C_{2}\right)$ is the union of one or two slit tori fixed by the hyperelliptic involution. Let $T$ be one of these tori. We call the elliptic involution of a torus $T$ embedded into a flat surface of higher genus $X$ an elliptic involution that fixes one of the singularities of $X$ that lies on $T$.

Claim 3.7. Suppose that $T$ is a slit torus and that the direction of the slit does not contain a lattice vector of $\Lambda$ where $T \cong \mathbb{C} / \Lambda$. Then $T$ contains a metric cylinder that does not intersect the slit and that is invariant under the hyperelliptic involution. 
Proof of the Claim. Up to normalization, the lattice is

$$
\Lambda=\left\langle\left(\begin{array}{l}
1 \\
0
\end{array}\right),\left(\begin{array}{l}
x \\
y
\end{array}\right)\right\rangle
$$

and slit is vertical. By our hypothesis, we may assume $x \in(0,1)$. As shown in Figure2, the direction $\left(\begin{array}{l}x \\ y\end{array}\right)$ is periodic and the cylinder contains a Weierstrass point. Moreover, the periodic leaves of the cylinder do not intersect the slit.

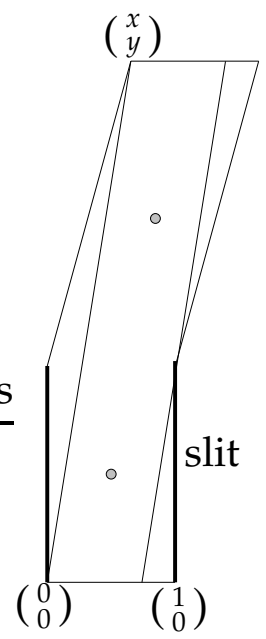

Figure 2. A cylinder fixed by the elliptic involution on a slit torus

The lengths of each slit is at least $L$ and the area of $T$ is less than 1 . Thus the distance between the left and right boundaries of $T$ is at most $1 / L$ and the length of the cylinder is at least $L$. This yields $|\cos (\theta)| \leq 1 / L^{2}$.

It remains to consider the case described in Figure 1 h when $X \backslash\left(C_{1} \cup C_{2}\right)$ is empty. There is a segment starting from the Weierstrass point which is in the middle of the saddle connection labelled by 3 to the middle of the saddle connection labelled by 5 . This segment and its image under the hyperelliptic involution form a closed geodesic $\gamma$ in a direction $\theta$. The periodic geodesic $\gamma$ is the core of a cylinder (containing a Weierstrass point) and the angle $\theta$ satisfies $|\cos (\theta)| \leq 1 / L^{2}$. This ends the proof of Lemma 3.6 .

Proof of Theorem 3.2. We assume that the area of $(X, \omega)$ is equal to one and we fix $\varepsilon>0$ and $\phi \in \mathbb{S}^{1}$. Applying Masur's theorem, there exists a periodic direction $\psi$ with $|\psi-\phi|<\varepsilon / 2$. On the other hand, for each $\delta$, there is only finite number of directions with a saddle connection of length less than $\delta$. We may thus assume, 
without loss of generality, that the length of the shortest saddle connection in the direction $\psi$ is at least $\frac{1}{\sqrt{\sin (\varepsilon / 2)}}$. By Lemma 3.6, there is a periodic direction $\theta$ with a cylinder containing a Weierstrass point and $|\sin (\theta-\psi)|<\sin (\varepsilon / 2)$ for $\varepsilon$ small enough. Thus $|\phi-\theta|<\varepsilon$. Theorem 3.2 is proven.

3.2. Proof of Theorem 3.3 . We first recall some facts concerning the J-invariant of Kenyon-Smillie [KS00] and the SAF-invariant.

Theorem 3.8 (Calta-Smillie [CS07] Theorem 1.5). Assume that $(X, \omega)$ is stabilized by a pseudo-Anosov diffeomorphism and that there exists a completely periodic direction. Then the SAF-invariant vanishes for all directions of the trace field.

The following facts concerning the SAF-invariant are essentially contained in Arnoux's thesis [Arn81] and were used by Calta [Cal04] and also by McMullen [Mc2] (who works with the flux instead of the SAF-invariant).

Lemma 3.9. Let $(Y, \alpha)$ be a translation surface in the stratum $\mathcal{H}(2)$ such that the SAFinvariant vanishes in the vertical direction. Then the vertical direction is completely periodic.

Lemma 3.10. Let $(Y, \alpha)$ be a translation surface in the stratum $\mathcal{H}(1,1)$ such that the $S A F$-invariant vanishes in the vertical direction. If there are two non homologous vertical saddle connections, then the vertical direction is completely periodic.

Proof of Lemmas 3.9, 3.10, For the sake of the completeness, we give a sketch of proof of Lemmas 3.9, 3.10, We recall that, in genus 2, if the SAF-invariant vanishes then the flow in the vertical direction is not minimal [Ar]. Therefore, there is a vertical saddle connection.

If $(Y, \alpha)$ belongs to $\mathcal{H}(2)$, the induced map of the vertical flow on a suitable transversal is an interval exchange transformation on 4 intervals. Since there is a saddle connection, it reduces to an interval exchange transformation on 3 intervals. An interval exchange on 3 intervals with SAF-invariant equals to 0 is periodic (see [Arn81]). Thus, the vertical flow on $(Y, \alpha)$ is periodic.

If $(Y, \alpha)$ belongs to $\mathcal{H}(1,1)$, the induced map of the vertical flow on a suitable transversal $\tau$ is an interval exchange transformation on 5 intervals. By hypothesis, there are two vertical saddle connections $\gamma_{1}$ and $\gamma_{2}$ such that $Y \backslash\left(\gamma_{1} \cup \gamma_{2}\right)$ is connected. Thus, the induced map on $\tau$ reduces to an interval exchange transformation on 3 intervals with SAF-invariant equals to 0 . This interval exchange transformation is periodic and thus vertical flow is periodic.

Proof of Theorem 3.3. Let us consider a cylinder $C$ fixed by the hyperelliptic involution on $(X, \omega)$. Let $\theta$ be the direction of $C$. It is a direction of the holonomy 
field (see background) thus, by Calta-Smillie's result, the SAF-invariant vanishes in the direction $\theta$. Without loss of generality we may assume that the direction $\theta$ is the vertical direction. The complement of $C$ is a slit surface $Y$ with vertical boundaries. We have to prove that the vertical flow is completely periodic on $Y$. The SAF-invariant equals to 0 on $C$, thus it vanishes on $Y$. The topological type of $Y$ can be deduced from the description of the completely periodic directions (see Figure 1) by the same argument as in Lemma 3.6.

There is a canonical way to associate to $Y$ a compact surface $\hat{Y}$ without boundary. $Y$ is obtained from $X$ by removing a cylinder $C$ invariant under the hyperelliptic involution. A slit on $Y$ corresponds to a saddle connection on the boundary of $C$ and that is not invariant under the hyperelliptic involution. Since $C$ is fixed by the hyperelliptic involution, these slits come in pairs. $\hat{Y}$ is obtained from $Y$ by gluing the pairs of slits exchanged by the hyperelliptic involution. The vertical flow on $Y$ is periodic if and only if it is periodic on $\hat{Y}$.

Referring to the Figure 1, we denote the cylinders by I, II, III, etc from the left to the right.

- $\hat{Y}$ belongs to $\mathcal{H}(2)$, if II is removed in Figure1a.

- $\hat{Y}$ belongs to $\mathcal{H}(2)$, if II is removed in Figure $1 \mathrm{~b}$.

- $\hat{Y}$ belongs to $\mathcal{H}(1,1)$, if I is removed in Figure $1 \mathrm{c}$.

- $\hat{Y}$ belongs to $\mathcal{H}(1,1)$, if II is removed in Figure 1 k.

- $\hat{Y}$ belongs to $\mathcal{H}(1,1)$, if III is removed in Figure $1 c$.

- $\hat{Y}$ belongs to $\mathcal{H}(2)$, if I is removed in Figure $1 \mathrm{~d}$.

- $\hat{Y}$ belongs to $\mathcal{H}(2)$, if II is removed in Figure $1 \mathrm{~d}$.

- $\hat{Y}$ is the union of two cylinders, if III is removed in Figure 1d.

- $\hat{Y}$ belongs to $\mathcal{H}(2)$, if II is removed in Figure1e.

- $\hat{Y}$ belongs to $\mathcal{H}(2)$, if IV is removed in Figure 1 e.

- $\hat{Y}$ belongs to $\mathcal{H}(2)$, if II is removed in Figure 1f.

- $\hat{Y}$ belongs to $\mathcal{H}(2)$, if IV is removed in Figure $1 \mathrm{f}$.

- $\hat{Y}$ belongs to $\mathcal{H}(2)$, if IV is removed in Figure $1 \mathrm{~g}$.

- $\hat{Y}$ belongs to $\mathcal{H}(2)$, if II is removed in Figure $1 \mathrm{~g}$.

- $\hat{Y}$ belongs to $\mathcal{H}(2)$, if I is removed in Figure 1 i.

- $\hat{Y}$ is a cylinder, if II is removed in Figure 1

- $\hat{Y}$ belongs to $\mathcal{H}(1,1)$ if I is removed in Figure 1 .

- $\hat{Y}$ is a cylinder, if II is removed in Figure 1]

- $\hat{Y}$ belongs to $\mathcal{H}(2)$, if I is removed in Figure $1 \mathrm{k}$.

- $\hat{Y}$ belongs to $\mathcal{H}(2)$, if II is removed in Figure $1 k$. 
By Lemma 3.9, the vertical direction is completely periodic when $\hat{Y} \in \mathcal{H}(2)$. We have to check the hypothesis of Lemma 3.10 when $\hat{Y}$ belongs to $\mathcal{H}(1,1)$. We treat the case where the cylinder I is removed in Figure $1 k$. The analysis in the other cases is more or less the same. The surface $Y$ contains two vertical saddle connections isometric to the saddle connections labelled by 1 and 2 in Figure 1 c. These connections don't disconnect the surface thus, by Lemma 3.10, the flow in the vertical direction is completely periodic on $\hat{Y}$. Therefore the vertical direction is completely periodic on $(X, \omega)$.

Combining Theorem 3.2 and the previous argument, we immediately deduce that there is a dense set of completely periodic directions on $(X, \omega)$. The theorem is proven.

\section{Detecting the Closure: Reduction Steps}

In this section we show in several instances that a completely periodic direction on a surface $(X, \omega)$ plus some irrationality condition implies the existence of a more useful completely periodic direction on a surface $(Y, \eta)$ in the $\mathrm{GL}_{2}^{+}(\mathbb{R})$-orbit closure of $(X, \omega)$. Moreover, $(Y, \eta)$ can be chosen such that the new completely periodic direction still satisfies some irrationality condition. The underlying strategy of all lemmas in this section is roughly the same. Finally, we arrive in a situation where we can show that the $\mathrm{GL}_{2}^{+}(\mathbb{R})$-orbit closure of $(Y, \eta)$ is all of $\mathcal{L}$.

A bit more precisely, we start with a direction $p$ on $(X, \omega)$ for which there exists a splitting of the surface into some cylinders and/or tori. We apply cyclic unipotent group $U$ (with eigenvector $p$ ) on $(X, \omega)$. This action stabilizes globally the splitting and acts on the product of tori and/or cylinders. We then apply Ratner's theorem on orbit closures of the cyclic unipotent group $U$ inside $U^{k}$ or $\mathrm{SL}_{2}(\mathbb{R})^{k}$ for $k=2,3$. The orbit closure is of the form $H \cdot(X, \omega)$, where $H$ is a closed subgroup of $\mathrm{SL}_{2}(\mathbb{R})^{k}$ for $k=2,3$, in the closure of the $\mathrm{SL}_{2}(\mathbb{R})$-orbit of $(X, \omega)$. Then with "generic"

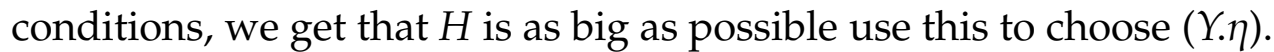

Two types of splitting directions will be particularly useful. The first one is a 3C-direction; this is a completely periodic direction with three cylinders and a configuration of saddle connections as the vertical direction of Figure 1 d. Of course, this is not the only direction with three cylinders, but we stick to this short notation also used in [HLM06]. The second one is defined as follows.

Definition 4.1. A $2 T_{\text {fix }} 2 C$-direction is a direction with two simple cylinders exchanged by the hyperelliptic involution and such that the complement decomposes into two tori. The vertical direction in Figure 1e is an example, which is in fact completely periodic. A $2 T_{\text {fix }} 2 C$-direction is not always completely periodic. In fact if a $2 T_{\text {fix }} 2 \mathrm{C}$-direction is not completely periodic, the flow, in that direction, 
contains one or two minimal component(s), corresponding to the two tori. We will say that a $2 T_{\text {fix }} 2 C$-direction is irrational if it is not completely periodic.

Remark 4.1.1. We alert a reader who has also read [HLM06], that there such a direction was simply called $2 T 2 C$. But we need to distinguish $2 T_{\text {fix }} 2 C$-directions from $2 T 2 C_{\text {fix }}$-directions. A completely periodic $2 T 2 C_{\text {fix }}$-direction (e.g. in Figure 1f) has a two simple cylinders fixed by the hyperelliptic involution and, cutting along all saddle connections in that direction, the complement consists also of two components, exchanged by the hyperelliptic involution. These components can be glued in a obvious way to two tori. It will be convenient also to talk of irrational $2 T 2 C_{\text {fix }}$-directions, although this is some abuse of terminology. Such a direction (cf Figure $7 \mathrm{a}$ ) also has two simple cylinders fixed by the hyperelliptic involution, but no saddle connections in the complement. This complement is then connected and thus it is a surface of genus two with two slits (rather than two tori).

We now give some useful definitions for the upcoming lemma.

Definition 4.2. For any lattice $\Lambda$ we denote by $\Lambda^{\prime}$ the normalized area one lattice obtained by rescaling $\Lambda$ by a homothety. We say that two lattices $\Lambda_{1}$ and $\Lambda_{2}$ are strongly non-commensurable with respect to a direction $p \in \mathbb{R}^{2}$, if there is no unipotent element $u$ with eigenvector $p$ such that $u\left(\Lambda_{1}^{\prime}\right)$ and $\Lambda_{2}^{\prime}\left(\right.$ or $\Lambda_{1}^{\prime}$ and $\left.u\left(\Lambda_{2}^{\prime}\right)\right)$ are commensurable.

Definition 4.3. We say that 3 moduli $m_{i}$ of cylinders, in a completely periodic direction are pairwise incommensurable, if $m_{i} / m_{j} \neq \mathbb{Q}$ for $i \neq j$, i. e. if they are $\mathbb{Q}-$ linearly independent or admit a $\mathbb{Q}$-linear relation $\sum_{i=1}^{3} a_{i} m_{i}=0$ unique up to scalars and with $a_{i} \neq 0$ for all $i$.

The first lemma consists of the key argument of [HLM06]. We recall the proof in details. From Lemma 4.5 to Lemma 4.11 we will relax the hypothesis on the translation surface $(X, \omega)$.

Lemma 4.4. Let us assume that $(X, \omega) \in \mathcal{L}$ contains a $3 C$-direction. Then it contains also a transverse $2 T_{\text {fix }} 2 C$-direction, denoted by $p$ : it is given by the four homologous saddle connection drawn in Figure 3 Let us assume that the direction $p$ has irrational and strongly non-commensurable tori. Then the $\mathrm{GL}_{2}^{+}(\mathbb{R})$-orbit closure of $(X, \omega)$ is $\mathcal{L}$.

Proof. Let us denote by $Z$ the closure of $(X, \omega)$ under $\mathrm{SL}_{2}(\mathbb{R})$ inside $\mathcal{L}_{1}$, the real hypersurface of translation surfaces of $\omega$-volume one. One has to show that $Z=\mathcal{L}_{1}$. Note that $\mathcal{L}_{1}$ has real dimension 11 . Let $U$ the unipotent subgroup of $\mathrm{SL}_{2}(\mathbb{R})$ generated by unipotent elements $u$ having $p$ has eigenvector. Then the action of $U$ on $(X, \omega)$ is very simple: it stabilizes globally the direction $p$ and it acts on each component of the splitting. Thus $U$ acts on the space of a pair of tori 
and a cylinder, which is isomorphic to $\left(\mathrm{SL}_{2}(\mathbb{R}) / \mathrm{SL}_{2}(\mathbb{Z})\right)^{2} \times U$. Thanks to Ratner's theorem, the closure of $U \cdot(X, \omega)$ is algebraic, i. e. $H \cdot(X, \omega)$ where $H$ is a closed subgroup of $\mathrm{SL}_{2}(\mathbb{R})^{2} \times U$ containing $U$ diagonally embedded.

The hypothesis that $p$ is irrational and strongly non-commensurable implies that this splitting is "generic" for Ratner, i. e. $H=\mathrm{SL}_{2}(\mathbb{R})^{2} \times U$. In other words, the closure of the unipotent group orbit in that direction contains all surfaces with the ratios of the splitting pieces fixed. Note that the $2 T_{\text {fix }} 2 C$-direction is characterized by the four homologous saddle connections. Thus we have proved that $Z$ contains a subset of real dimension $(2 * 3+1)+2=9$ where $2 * 3$ stands for the dimension of $\mathrm{SL}_{2}(\mathbb{R})^{2}, 1$ for the dimension of $U$ and 2 for the connection vector of the four homologous saddle connections realizing the splitting. We catch the two missing dimensions as follows.

During the previous discussion, the ratios of the area of the tori by the area of the cylinder are fixed. Thus one has to vary these two ratios.

Let us consider the direction $\tilde{p}$ obtained by applying a simple Dehn twist around the vertical non-simple cylinder of the $3 C$-direction (see Figure 3 ). The direction $\tilde{p}$ is obviously again of type $2 T_{\text {fix }} 2 C$. Since the saddle connections of a $2 T_{\text {fix }} 2 C$-direction are homologous, the splitting in the direction $\tilde{p}$ still exists in a neighborhood $N$ of $(X, \omega)$.

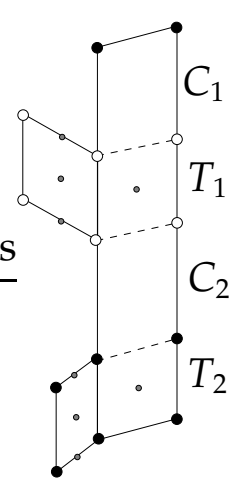

Figure 3. A "diagonal" resplitting $2 T_{\text {fix }} 2 C$-direction on a $3 C$-direction.

We write $(X, \omega)=T_{1} \# C_{1} \# T_{2} \# C_{2}$ to denote that $(X, \omega)$ is obtained as the connected sum of the $T_{i}$ and $C_{i}$ glued along a fixed set of slits, which is supressed in the notation. We then know that orbit closure of $(X, \omega)$ ontains $U T_{1} \# C_{1} \# U T_{2} \# C_{2}$, where $U$ is the unipotent vertical element. In particular for $\left(u_{1}, u_{2}\right)$ in a neighborhood of $(0,0)$ the twisted decomposition persists on $\left(X\left(u_{1}, u_{2}\right), \omega\left(u_{1}, u_{2}\right)\right):=U_{u_{1}} T_{1} \# C_{1} \# U_{u_{2}} T_{2} \# C_{2}$. Let us denote the splitting in the twisted direction by prime $\left(X^{\prime}\left(u_{1}, u_{2}\right), \omega^{\prime}\left(u_{1}, u_{2}\right)\right)=T_{1}^{\prime} \# C_{1}^{\prime} \# T_{2}^{\prime} \# C_{2}^{\prime}$. One can show 
(see [HLM06], Lemma 5.9) that $\left(u_{1}, u_{2}\right)$-twisting can indeed be used to adjust the areas. More precisely the map

$$
\varphi:\left(u_{1}, u_{2}\right) \mapsto\left(\operatorname{area}\left(T_{1}^{\prime}\left(u_{1}, u_{2}\right)\right) / \operatorname{area}\left(C_{1}^{\prime}\left(u_{1}, u_{2}\right)\right) \text {, area }\left(T_{2}^{\prime}\left(u_{1}, u_{2}\right)\right) / \operatorname{area}\left(C_{1}^{\prime}\left(u_{1}, u_{2}\right)\right)\right)
$$

is an invertible function in a neighborhood of $\left(u_{1}, u_{2}\right)=(0,0)$.

Thus for almost all $\left(u_{1}, u_{2}\right)$ in a neighborhood of $(0,0)$ with respect to the Lebesgue measure, we can apply Ratner's theorem in the new splitting direction. This provides us a subset $N \subset Z$, of positive measure, consisting of all above possible splitting with varying ratios. Recall that the geodesic flow is ergodic on the hyperelliptic locus $\mathcal{L}$ ([Mas82, Vee82]). Therefore $Z$ has full measure in $\mathcal{L}$ and the lemma is proven.

Lemma 4.5. Let us assume that $(X, \omega) \in \mathcal{L}$ contains an irrational $2 T_{\text {fix }} 2 C$-direction. In addition we assume that the two tori of the splitting, are strongly non-commensurable. Then the $\mathrm{GL}_{2}^{+}(\mathbb{R})$-orbit closure of $(X, \omega)$ is $\mathcal{L}$.

Proof. The idea is to apply previous Lemma 4.4. Let $p$ denote the direction of the $2 T_{\text {fix }} 2 C$-direction. As usual, let $U$ be the unipotent subgroup of $\mathrm{SL}_{2}(\mathbb{R})$ generated by unipotent elements $u$ having $p$ has eigenvector. By Ratner's theorem, in the $\mathrm{SL}_{2}(\mathbb{R})$ orbit closure of $(X, \omega)$, one finds surfaces $H \cdot(X, \omega)$ where $H$ is a closed unimodular subgroup of $\mathrm{SL}_{2}(\mathbb{R})^{2} \times U$. By hypothesis, $H$ contains at least the product $\mathrm{SL}_{2}(\mathbb{R})^{2}$. Thus one can apply a unipotent matrix in each torus independently, in order to obtain a surface $(Y, \eta)$ in the $\mathrm{SL}_{2}(\mathbb{R})$-orbit closure of $(X, \omega)$ with a $2 T_{\text {fix }} 2 C$-direction and an adequate transverse $3 C$-direction (cf Figure 3).

Of course, by construction, the tori of the $2 T_{\text {fix }} 2 C$-direction on $(Y, \eta)$ are still irrational. By modifying slightly $(X, \omega)$ by a diagonal matrix in $\mathrm{SL}_{2}(\mathbb{R})$ before applying Ratner's theorem, one can obtain $(Y, \eta)$ without destroying above properties and in addition two strongly non-commensurable tori. We then conclude by applying Lemma 4.4 .

Lemma 4.6. If $(X, \omega) \in \mathcal{L}$ contains an irrational $2 T_{\text {fix }} 2 C$-direction, then the $\mathrm{GL}_{2}^{+}(\mathbb{R})$-orbit closure of $(X, \omega)$ is $\mathcal{L}$.

Proof. The strategy is to use previous Lemma 4.5. We will prove that there exists a surface $(Y, \eta)$ in the $\mathrm{GL}_{2}^{+}(\mathbb{R})$-orbit closure of $(X, \omega)$ with an irrational $2 T_{\mathrm{fix}} 2 C$ direction containing two strongly non-commensurable tori.

$\mathrm{Up}$ to a rotation, we can and will assume that the $2 T_{\text {fix }} 2 C$-direction is vertical as in Figure 4. We apply the unipotent subgroup $\left(\begin{array}{ll}1 & 0 \\ * & 1\end{array}\right)$ to the vertical splitting. Exchanging the role of $T_{1}$ and $T_{2}$ if necessary, one can assume that $T_{1}$ is irrational. As in previous lemma, we denote the splitting $(X, \omega)=C_{1} \# T_{1} \# C_{2} \# T_{2}$. As usual, using Ratner's theorem one deduces that the $S_{2}(\mathbb{R})$-orbit closure of $(X, \omega)$ contains $C_{1} \# S L_{2}(\mathbb{R}) \cdot T_{1} \# C_{2} \# T_{2}$. Said differently, the closure contains all surfaces 

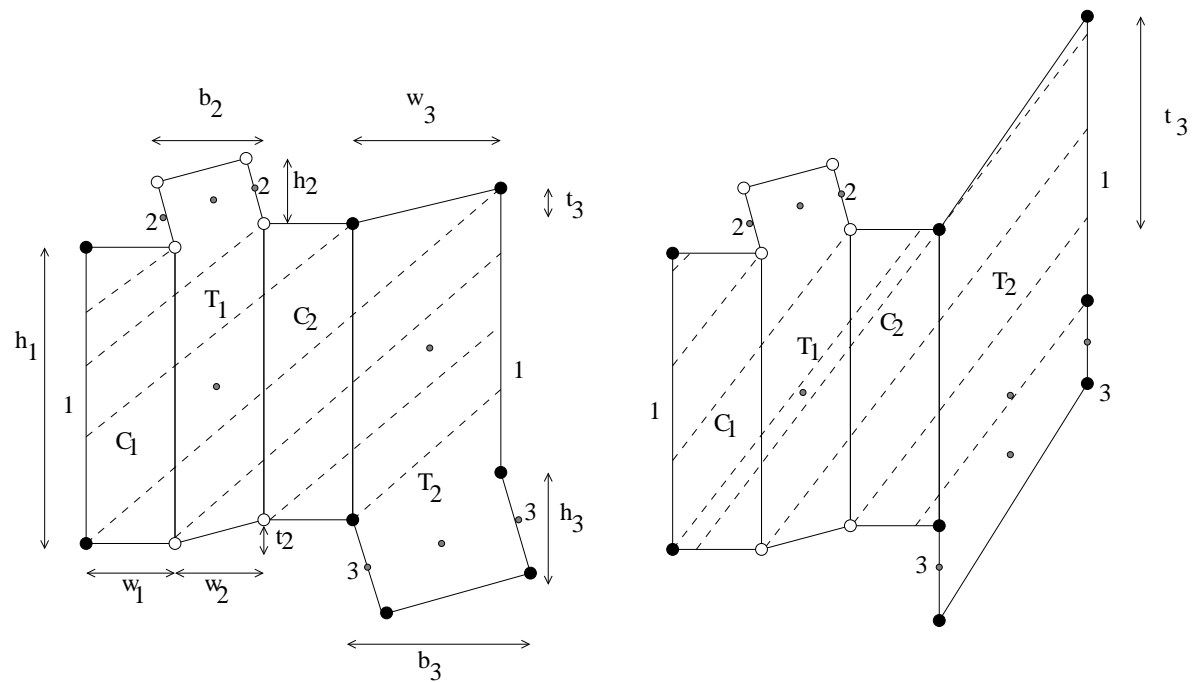

Figure 4. A $2 T_{\text {fix }} 2 \mathrm{C}$-direction with an irrational torus and a "diagonal" resplitting with $p>t_{3} / w_{3}$ on the left figure and $p<t_{3} / w_{3}$ on the right figure

with the following properties. All the parameters $w_{1}, w_{3}, h_{1}, h_{3}$ and $b_{3}$ are as given by the original surface $(X, \omega)$. Note that the torus $T_{2}$ is not necessarily periodic. If it is, one has $b_{3}=w_{3}$, as indicated in the right figure.

The parameters $w_{2}, t_{2}$ and $b_{2}$ can be chosen arbitrarily as long as $b_{2}>w_{2}$ and $h_{2}$ can be chosen to satisfy the volume condition $\left(h_{1}+h_{2}\right) \cdot w_{2}=A_{1}$ where $A_{1}$ is the volume of the irrational torus $T_{1}$ of $(X, \omega)$. In particular $w_{2}$ can be chosen close to 0 . By flipping the figure and changing the sign of $t_{2}$, we may suppose that both $t_{2}$ and $t_{3}$ are non-negative.

Let us denote the splitting in the new direction (prescribed by the dotted line) by prime. This new direction, by construction, crosses $k$-times the top of each vertical cylinder $C_{i}$ as indicated on the figure ( $k=0$ in Figure 4 on the left and $k=1$ on the right). This direction has slope

$$
p=\frac{(k+1) h_{1}+t_{2}+t_{3}}{2 w_{1}+w_{2}+w_{3}} .
$$

Moreover one can move parameters $w_{2}, t_{2}$ and $b_{2}$ in order to obtain a $2 T_{\text {fix }} 2 C$ direction for the new splitting by the following way. There are two cases to consider: $p>t_{3} / w_{3}$ (see the left figure) and for $p<t_{3} / w_{3}$ (see the right figure). The case of equality can be avoided by modifying $t_{2}$ slightly. Note that using the hyperelliptic involution it is enough to check the intersection behaviour for the 
dotted saddle connections emmanating from the white singularity on the bottom of $C_{2}$.

Choosing $w_{2}$ close to 0 and $t_{2} / w_{2}$ small enough, the line emanating at the white point has the correct intersection behaviour if and only if

$$
\frac{k h_{1}+t_{3}}{w_{1}+w_{3}}<p<\frac{(k+1) h_{1}+t_{3}}{w_{1}+w_{3}} .
$$

This condition can always be satisfied choosing $k$ suitably and equality can again be excluded by modifying $t_{2}$ slightly. Therefore the dotted line provides a new splitting $C_{1}^{\prime} \# T_{1}^{\prime} \# C_{2}^{\prime} \# T_{2}^{\prime}$.

Thus, until now, we have found a resplitting in a $2 T_{\text {fix }} 2 C$-direction for all $t_{2}, w_{2}$ and $h_{2}$ in a small open intervals. We will check that for almost all triples $\left(t_{2}, w_{2}, b_{2}\right)$, subject to the condition that $\left(h_{1}+h_{2}\right) w_{2}=A_{1}$ is fixed, this splitting satisfies the conditions of Lemma 4.5. Namely, for almost all triples the two new tori $T_{1}^{\prime}$ and $T_{2}^{\prime}$ are irrational and strongly non-commensurable.

We give detailed proof for the left figure case; the right figure case being completely similar. Thus the lattices of the tori (for the resplitting $2 T_{\text {fix }} 2 C$-direction) are

and

$$
\Lambda_{1}^{\prime}=\left\langle v_{1}^{\prime}:=\left(\begin{array}{c}
w_{2} \\
t_{2}
\end{array}\right), w_{1}^{\prime}:=\left(\begin{array}{c}
2 w_{2}-b_{2}+2 w_{1}+w_{3} \\
(k+1) h_{1}+h_{2}+t_{2}+t_{3}
\end{array}\right)\right\rangle
$$

$$
\Lambda_{2}^{\prime}=\left\langle v_{2}^{\prime}:=\left(\begin{array}{c}
w_{3} \\
t_{3}
\end{array}\right), w_{2}^{\prime}:=\left(\begin{array}{c}
2 w_{3}-b_{3}+2 w_{1}+w_{2} \\
(k+1) h_{1}+h_{3}+t_{2}+t_{3}
\end{array}\right)\right\rangle .
$$

One has to make sure that these two lattices do not possess vectors in the direction of $p$. Moreover we want that $\Lambda_{1}$ and $\Lambda_{2}$ are strongly non-commensurable with respect to $p$ in order to apply previous lemma. A direct calculation shows that if $\Lambda_{1}$ (respectively $\Lambda_{2}$ ) possesses vectors in the direction of $p$ then there exists $n \in \mathbb{Z}$ such that

$$
\frac{n t_{2}+h_{2}}{(k+1) h_{1}+h_{3}} \in \mathbb{Q} \quad\left(\text { respectively } \frac{n t_{3}+h_{3}}{(k+1) h_{1}+h_{2}} \in \mathbb{Q}\right) .
$$

One gets similar conditions for the strong non-commensurability of the lattices. These two conditions exclude only a 2-dimensional set of Lebesque measure (in $w_{2}, t_{2}, h_{2}$ and $b_{2}$ with $\left.\left(h_{1}+h_{2}\right) w_{2}=A_{1}\right)$ equals to zero. Therefore we have found a resplitting in a $2 T_{\text {fix }} 2 C$-direction, for almost all $t_{2}, w_{2}$ and $h_{2}$, with two irrational and strongly non-commensurable tori. We then conclude the proof of Lemma 4.6 by using Lemma 4.5 .

Lemma 4.7. If $(X, \omega)$ has a $3 C$-direction with incommensurable moduli, the $\mathrm{SL}_{2}(\mathbb{R})$ orbit closure of $(X, \omega)$ is $\mathcal{L}$. 
Proof. If $p$ denotes the $3 C$-direction, let $U$ be the subgroup of $\mathrm{SL}_{2}(\mathbb{R})$ generated by unipotent elements with eigenvector $p$. We will also denote by $\tilde{p}$ the transversal $2 T_{\text {fix }} 2 \mathrm{C}$-direction (see Figure 3 ). We use Ratner's theorem for $U$ on the space of triples of normalized lattices $\left(\mathrm{SL}_{2}(\mathbb{R}) / \mathrm{SL}_{2}(\mathbb{Z})\right)^{3}$ to ensure that the direction $\tilde{p}$ possesses an irrational torus. Then we conclude by using Lemma 4.6

In order to prove similar lemmas for other completely periodic directions, incommensurability is not strong enough. This will be clear from the covering constructions in Section [7, In Section [5] we prove that if $(X, \omega)$ is a pseudo-Anosov surface and if the trace field is totally real and has degree 3 over $\mathbb{Q}$ then any non-parabolic completely periodic direction with 3 moduli of cylinders is pairwise incommensurable. (Corollary 5.2). Hence, in the following lemmas, we can replace "pairwise incommensurable moduli" by "non-parabolic" for surfaces arising from Thurston's construction.

Lemma 4.8. If $(X, \omega)$ has a completely periodic $2 T_{\text {fix }} 2 C$-direction with pairwise incommensurable moduli, then the $\mathrm{GL}_{2}^{+}(\mathbb{R})$-orbit closure of $(X, \omega)$ is $\mathcal{L}$.

Proof. Using the action of $\mathrm{SL}_{2}(\mathbb{R})$ we may suppose that the $2 T_{\text {fix }} 2 \mathrm{C}$-direction is vertical and that the bottom of the cylinders $C_{i}$ is horizontal. Suppose that the saddle connections of slope $p:=\left(h_{1}+t_{2}+t_{3}\right) /\left(2 w_{1}+w_{2}+w_{3}\right)$ intersect the vertical saddle connections as drawn in Figure 5. Then the direction of slope $p$ is again

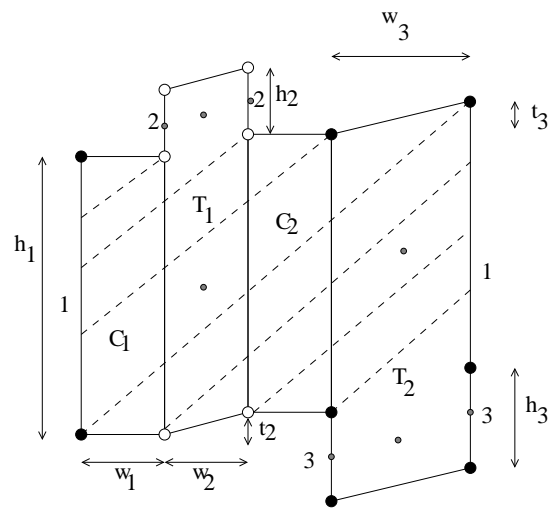

Figure 5. Resplitting of a $2 T_{\text {fix }} 2 C$-direction

$2 T_{\text {fix }} 2 \mathrm{C}$ and we want to make sure that, say, the torus containing the top of $T_{2}$ is not periodic. Sliding the Weierstrass points along the slope $p$ we observe that this unwanted property holds if

$$
\frac{h_{2}+h_{1}}{2 p}-\frac{2 w_{1}+w_{2}+w_{3}}{2} \in \mathbb{Q} w_{3} .
$$


Equivalently, we have to ensure that $t_{3}+t_{2}$ does not lie in a coset of $\mathbb{Q}$ in $\mathbb{R}$. By hypothesis the orbit closure of $(X, \omega)$ under the vertical unipotents contains a oneparameter subgroup that fixes $C_{1}$ and hence $C_{2}$. Let $m_{1}=w_{1} /\left(h_{1}+h_{2}\right)$ and $m_{2}=$ $w_{2} /\left(h_{1}+h_{3}\right)$ be the modulus of $T_{1}$ and $T_{2}$ respectively. Simple cylinders persist under small deformations. For $u \in \mathbb{R}$ small enough, we may thus replace $t_{1}$ by $t_{1}+u$ and $t_{2}$ by $t_{2}+u m_{2} / m_{1}$ to obtain a surface in the unipotent orbit closure of $(X, \omega)$ still with a $2 T_{\text {fix }} 2 C$-direction. Since $m_{2} / m_{1}$ is irrational by pairwise incommensurabilty, we can choose $u$ in order to avoid the unwanted coset. We can now apply Lemma 4.6.

It remains to check that one can always choose $t_{2}$ and $t_{3}$ with the desired intersection property. This property is surely satisfied for both $t_{2}$ and $t_{3}$ close to zero. Recall that a twist $t_{2}$ (resp. $t_{3}$ ) is only well-defined up to integer multiples $h_{1}+h_{2}$ (resp. $\left.h_{1}+h_{3}\right)$. It suffices to find the resplitting not on $(X, \omega)$ but on a surface in the orbit closure of vertical unipotents. Hence it suffices to find $u \in \mathbb{R}$ such that

$$
u w_{2}+t_{2} \bmod h_{1}+h_{2} \text { and } u w_{3}+t_{3} \bmod h_{1}+h_{3}
$$

are both close to zero. Such $u$ exist since $m_{1} / m_{2}$ is irrational by pairwise incommensurability.

Lemma 4.9. If $(X, \omega)$ has a completely periodic $2 T 2 C_{\mathrm{fix}}$-direction (as in Figure 1f or Figure 6 (a) below) and such that the moduli $m_{i}$ of the cylinders $\left\{C_{1}, C_{2}, T_{1}\right\}$ are pairwise incommensurable then the $\mathrm{GL}_{2}^{+}(\mathbb{R})$-orbit closure of $(X, \omega)$ is $\mathcal{L}$.

Proof. We distinguish two cases. Suppose the $2 T 2 C_{\text {fix }}$-direction is vertical as in Figure 6(a). We first treat the case $h_{2}=h_{3}$ as suggested by the figure. This is quite

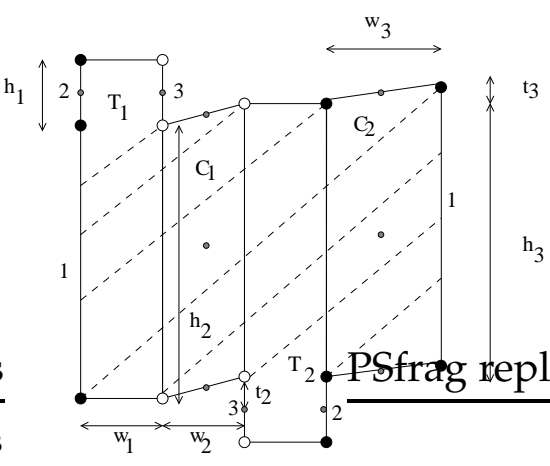

(a)

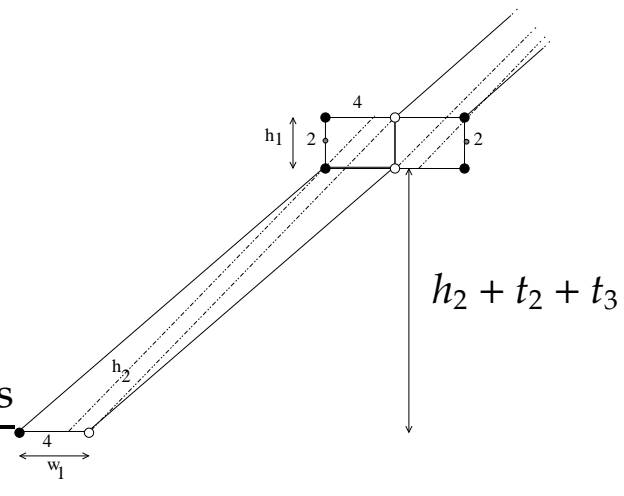

(b)

Figure 6. Resplitting of a $2 T 2 C_{\text {fix }}$-direction with $h_{2}=h_{3}$

analogous to the case of a $2 T 2 C_{\text {fix }}$-direction: Using pairwise incommensurability we may suppose that $t_{2}$ and $t_{3}$ are both close to zero such that the indicated splitting 
exists. Instead of resplitting in the direction of a 'simple Dehn twist around the almost-cylinder of height $h_{2}{ }^{\prime}$ we may also use a $k$-fold Dehn twist, for $t_{2}$ and $t_{3}$ even closer (depending on $k$ ) to zero. All these resplitting directions are irrational $2 T 2 C_{\text {fix }}$-directions and the complement of the two cylinders looks as in Figure 6 (b). The dotted lines have slope

$$
\tilde{p}=\frac{h_{2}+t_{2}+t_{3}}{k\left(2 w_{1}+w_{2}+w_{3}\right)-w_{1}} .
$$

If for some $m$

$$
\frac{h_{1}}{2 m w_{1}}>\tilde{p}>\frac{h_{1}}{(2 m+1) w_{1}},
$$

the direction of the dotted lines have 4 homologous saddle connections. Consequently $\tilde{p}$ is a a $2 T_{\text {fix }} 2 C$-direction, as illustrated in Figure 7 (a).

If we let $w:=2 w_{1}+w_{2}+w_{3}$, the above condition for $\tilde{p}$ may be rephrased as

$$
\frac{k}{2 m} \frac{w h_{1}}{w_{1}}>h_{2}+t_{2}+t_{3}+\frac{h_{1}}{2 m w_{1}} \text { and } h_{2}+t_{2}+t_{3}+\frac{h_{1}}{(2 m+1) w_{1}}>\frac{k}{2 m+1} \frac{w h_{1}}{w 1} .
$$

If we choose $k / 2 m$ close to $h_{2} w_{1} / w h_{1}$ and $m$ large enough, the inequalities are satisfied for $t_{2}$ and $t_{3}$ small enough. This and the existence of the $k$-fold Dehn twist for the chosen $k$ given two bounds for the size of $t_{2}$ and $t_{3}$.

If $t_{2}$ and $t_{3}$ chosen below both bounds and sufficiently irrational, the direction $\tilde{p}$ is an irrational $2 T_{\text {fix }} 2 C$-direction. Now Lemma 4.6 applies.

We now treat the case that $h_{2} \neq h_{3}$, say $h_{2}>h_{3}$. Consider the resplitting of Figure 7. This is a $2 T_{\text {fix }} 2 C$-direction $p$. Using incommensurability and the unipotent action in the vertical direction we may pass to an element in the unipotent orbit closure of $(X, \omega)$ and adjust $t_{2}$ to any suitable value, preserving the bottom of $T_{1}$ horizontal. The parameter $t_{3}$ will change, too, but this doesn't matter. We can find $t_{2}$ such that the torus in the direction $p$ whose intersection with $C_{2}$ is empty, is irrational. Lemma 4.6 applies.

Lemma 4.10. If $(X, \omega)$ has a completely periodic direction as in Figure $1 \mathrm{~g}$ or Figure 8 below and such that the moduli $m_{i}$ of the cylinders $\left\{C_{1}, C_{2}, C_{3}\right\}$ are pairwise incommensurable then the $\mathrm{GL}_{2}^{+}(\mathbb{R})$-orbit closure of $(X, \omega)$ is $\mathcal{L}$.

Proof. We may suppose that the completely periodic direction is vertical. The unipotent orbit closure of $(X, \omega)$ contains all surfaces as in Figure 8 , where the bottom of $C_{1}$ is horizontal and $t_{2}$ can be chosen arbitrarily. The resplitting give by the dotted lines is a $2 T_{\text {fix }} 2 C$-direction $p$. For suitable choice of $t_{2}$ the torus in the direction $p$ that does not intersect $C_{3}$ is irrational. We can now apply Lemma 4.6 . 


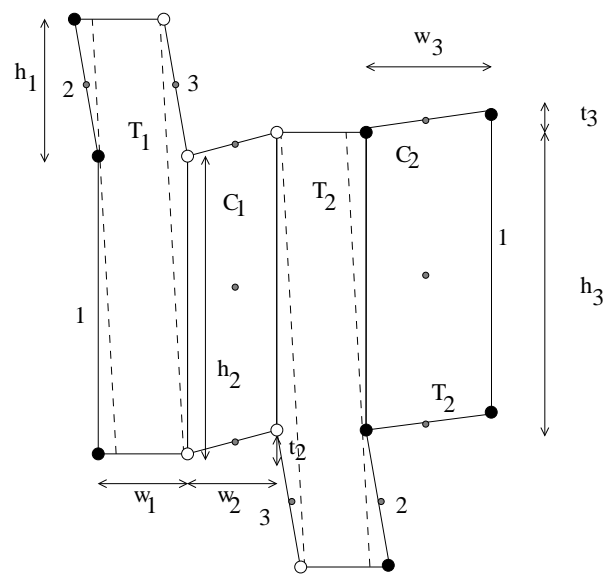

(a)

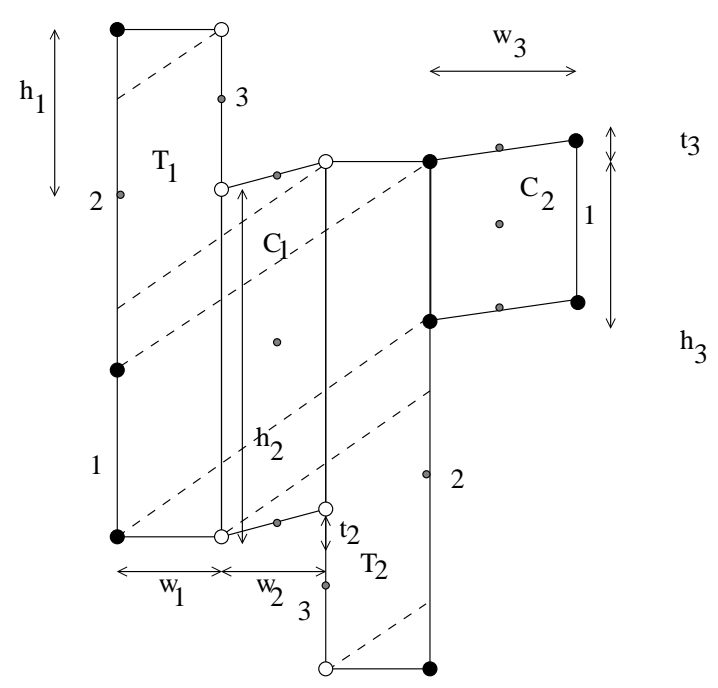

(b)

FIgURE 7. (a) $2 T_{\text {fix }} 2 C$-direction on an irrational $2 T 2 C_{\text {fix }}$-direction and (b) resplitting of a $2 \mathrm{~T} 2 C_{\mathrm{fix}}$-direction with $h_{2} \neq h_{3}$.

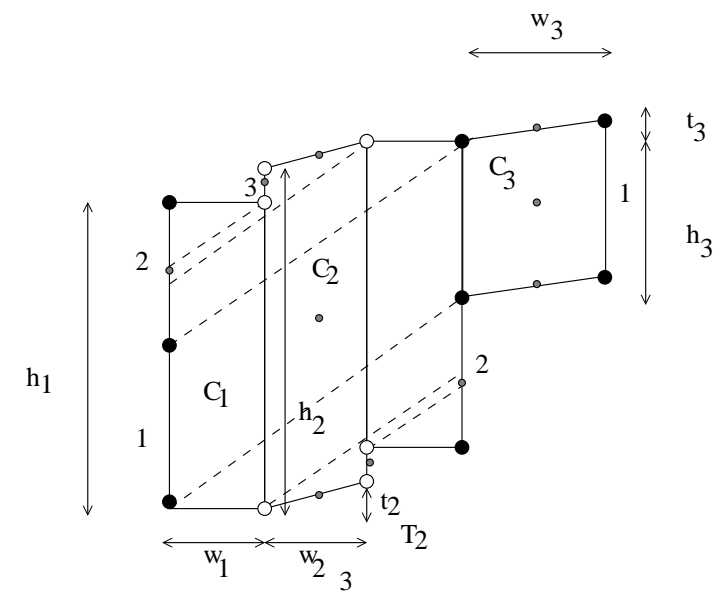

FIGURE 8 . Resplitting of a direction with 4 cylinders only one of which is simple

Lemma 4.11. If $(X, \omega)$ has a completely periodic direction as in Figure 15 or Figure 9 below and such that the moduli $m_{i}$ of the three cylinders are pairwise incommensurable, then the $\mathrm{GL}_{2}^{+}(\mathbb{R})$-orbit closure $(X, \omega)$ is $\mathcal{L}$. 
Proof. Suppose the periodic direction is vertical as in Figure 9. Using a vertical

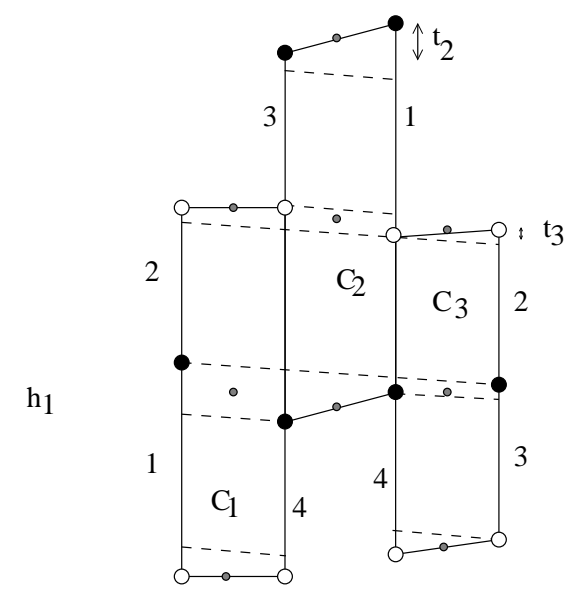

FigURE 9. Resplitting of a direction with 3 fixed cylinders

unipotent, we may suppose that $C_{1}$ is untwisted, i.e. has a horizontal saddle connection. For $t_{2}$ and $t_{3}$ sufficiently small but non-zero, the resplitting by dotted lines in Figure 9 is a $2 T_{\text {fix }} 2 C$-direction $p$. Moreover, for $t_{2}+t_{3}$ outside a coset of $\mathbb{Q}$ in $\mathbb{R}$ the direction $p$ has an irrational torus. We can arrange both conditions for a surfaces in the vertical unipotent orbit closure of $(X, \omega)$ by the same two arguments as in Lemma 4.8,

\section{SURFACES ARISING FROM THURSTON'S CONSTRUCTION}

In the whole section $(X, \omega)$ will be a surface with two transversal parabolic directions belonging to the hyperelliptic locus $\mathcal{L}$. We prove that if $(X, \omega)$ is not a Veech surface for the most obvious reason and if the pseudo-Anosov diffeomorphism is of a type that can only arise for $g \geq 3$, then its $\mathrm{GL}_{2}^{+}(\mathbb{R})$ orbit closure is large.

Theorem 5.1. Let $(X, \omega) \in \mathcal{L}$ be a surface given by Thurston's construction with trace field of degree 3. By Theorem 3.3 this surface has infinitely many completely periodic directions. Suppose that one of them is not parabolic. Then

$$
\overline{\mathrm{GL}_{2}^{+}(\mathbb{R}) \cdot(X, \omega)}=\mathcal{L} .
$$

We first study the properties of the moduli of cylinders for a non parabolic completely periodic direction. We then prove the theorem at the end of this section. 
5.1. Commensurability. Let $(X, \omega)$ be surface given by Thurston's construction. We recall that the affine group $\operatorname{SL}(X, \omega)$ of $(X, \omega)$ contains a pseudo-Anosov diffeomorphism $\varphi$ with trace field $K$ and a parabolic element $\rho$. Thanks to [HLa06] the field $K$ of $(X, \omega)$ is totally real. Let us assume that $[K: \mathbb{Q}]=3$. Let $\sigma_{i}: K \rightarrow \mathbb{R}$ be the different real embeddings. We fix one of them once and for all and for given $c \in K$ we shortly write $c^{\prime}:=\sigma_{2}(c)$ and $c^{\prime \prime}:=\sigma_{3}(c)$. Choose $\tau_{1}, \tau_{2} \in \operatorname{Gal}(\mathbb{R} / \mathbb{Q})$ such that

$$
\tau_{1} \circ \sigma_{1}=\sigma_{2} \text { and } \tau_{2} \circ \sigma_{1}=\sigma_{3}
$$

and such that $\tau_{1}$ have order 3 when restricted to a Galois closure of $K / \mathbb{Q}$.

Suppose that the horizontal direction of $(X, \omega)$ is completely periodic with cylinders of height $h_{i}$, circumference $c_{i}$ and moduli $m_{i}:=h_{i} / c_{i}$.

Lemma 5.2. Suppose the horizontal direction has $s=3$ or $s=4$ cylinders, such that two of them, say the third and the fourth, are interchanged by the hyperelliptic involution. Then either

i) the direction is parabolic, i.e. $m_{i} / m_{j} \in \mathbb{Q}$ for all $(i, j)$, or

ii) the moduli are $\mathbb{Q}$-linearly independent, or

iii) the moduli are related by

$$
\sum_{i=1}^{3} a_{i} m_{i}=0, \text { where } a_{i} \in \mathbb{Q}^{*},
$$

i.e. the moduli are not $\mathbb{Q}$-linearly independent but $m_{i} / m_{j} \notin \mathbb{Q}$ for $i \neq j$. In the terminology introduced in the previous section, the direction is either parabolic or pairwise incommensurable.

Proof. Applying an upper triangular unipotent to $(X, \omega)$ we may suppose that the relative periods are in $K(i)$. Let $t:=\operatorname{tr} \varphi$ and let

$$
\psi:=\varphi^{*}+\left(\varphi^{*}\right)^{-1} \in \operatorname{End}\left(H^{1}(X, \mathbb{R})\right) .
$$

By hypothesis on the trace field we have a decomposition into eigenspaces of $\psi$

$$
H^{1}(X, \mathbb{R})=S \oplus S^{\prime} \oplus S^{\prime \prime}
$$

where we number the eigenspaces such that $S=\langle\operatorname{Re}(\omega), \operatorname{Im}(\omega)\rangle$ and $\tau_{1}(S)=S^{\prime}$, $\tau_{2}(S)=S^{\prime \prime}$. Since $\psi$ is symplectic (see [Mc1] Theorem 7.1 and [Mc2] Theorem 9.7), the eigenspaces are orthogonal with respect to the cup product. The surface $(X, \omega)$ is covered by the cylinders and we conclude

(1) $\sum_{i=1}^{s} m_{i} c_{i} c_{i}^{\prime}=\sum_{i=1}^{s} h_{i} c_{i}^{\prime}=\int_{X} \operatorname{Re}(\omega) \wedge \operatorname{Im} \tau_{1}(\omega)=\frac{i}{4 \pi} \int_{X}(\omega+\bar{\omega}) \wedge\left(\tau_{1}(\omega)-\overline{\tau_{1}(\omega)}\right)=0$ 
Replacing $\tau_{1}$ by $\tau_{2}$ we similarly have

$$
\sum_{i=1}^{s} m_{i} c_{i} c_{i}^{\prime \prime}=0
$$

Moduli and circumferences of the cylinders exchanged by the hyperelliptic involution are the same. We apply $\tau_{2}$ to this equation and subtract it from the first one to obtain

$$
\sum_{i=1}^{3}\left(m_{i}-\tau_{2}\left(m_{i}\right)\right) \delta_{i} c_{i} c_{i}^{\prime \prime}=0,
$$

where $\delta_{i}=1$, except for the case of 4 cylinders where $\delta_{3}=2$. Suppose that the lemma is wrong, i. e. there is a relation $a_{1} m_{1}+a_{2} m_{2}=0$. Applying a matrix in $\mathrm{SL}_{2}(K)$ to $(X, \omega)$, we may suppose that $m_{3}$ is rational without changing the ratios of the $m_{i}$. We deduce from the above equations $m_{i}=\tau_{2}\left(m_{i}\right)$ for $i=1,2$. Hence in fact all $m_{i} \in \mathbb{Q}$ and we are in case i).

5.2. Completely periodic but not parabolic directions. The next lemma follows from Thurston's construction.

Lemma 5.3. Let $(X, \omega)$ be a surface given by Thurston's construction. If $(X, \omega)$ admits a parabolic direction with a decomposition into $k$ cylinders, then $[K: \mathbb{Q}] \leq k$. In particular, if $(X, \omega)$ admits a one cylinder decomposition then it is an arithmetic surface.

This lemma, combined with Lemma 5.2, gives together

Lemma 5.4. Let $(X, \omega) \in \mathcal{L}$ be a surface given by Thurston's construction with a completely periodic direction with two cylinders (or with three cylinders, two of which interchanged by the hyperelliptic involution). Then $[K: \mathbb{Q}] \leq 2$.

Proof. We discuss the case of two cylinders, the other case is similar since the exchanged cylinders have the same heights and widths. The equations (1) and (2) with $s=2$ hold in this situation, too. They yield

$$
\frac{m_{1}}{m_{2}}=-\frac{c_{2} c_{2}^{\prime \prime}}{c_{1} c_{1}^{\prime \prime}}=-\frac{c_{2} c_{2}^{\prime}}{c_{1} c_{1}^{\prime}}
$$

In particular $\tau_{1}\left(m_{1} / m_{2}\right)=m_{1} / m_{2}$. Therefore $m_{1} / m_{2} \in \mathbb{Q}$ and the horizontal direction is a parabolic direction with 2 cylinders. Applying Lemma [5.3 we get $[K: \mathbb{Q}] \leq 2$, which ends the proof.

In particular, we have:

Corollary 5.5. Let $(X, \omega) \in \mathcal{L}$ be a surface given by Thurston's construction with $[K$ : $\mathbb{Q}]=3$. The configuration of every completely periodic direction belongs to Figure 1k-111k-11f-1 $\mathrm{g}$. 
5.3. Proof of Theorem 5.1. By Lemma 5.4 the completely periodic direction has at least 3 orbits of cylinders under the hyperelliptic involution. By Theorem 2.1 and Corollary 5.5, it is thus one of the directions in Figure 1k or 1 d, Figure 1e or 1f, or Figure 1g. The Lemmas 4.11, 4.7, 4.8, 4.9 and 4.10, respectively, show that, if the direction is not parabolic, the orbit closure is as big as claimed. The hypothesis on pairwise incommensurability is met because of Lemma 5.2 .

\section{EXAMPLES}

In this section we show that Theorem 5.1 applies to infinitely many surfaces.

Theorem 6.1. There exist infinitely many surfaces given by Thurston's construction, with trace field of degree 3 , and with a non parabolic periodic direction.

We first construct such a surfaces (Lemma 6.3), and we then prove that they possess a completely periodic $2 T_{\text {fix }} 2 C$-direction, which is not parabolic (Proposition 6.5).

Proposition 6.2. Let $n$ be any positive integer and let $P=P_{n}$ be the polynomial $X^{3}-$ $2\left(n^{2}+3\right) X^{2}+\left(7 n^{2}+4\right) X-4 n^{2}$. Then $P$ possesses only real roots. If $\alpha=\alpha_{n}$ is the largest root, then $\alpha>1$.

Let us define the vectors $V=\left(V_{1}, V_{2}, V_{3}\right)$ and $H=\left(H_{1}, H_{2}, H_{3}\right)$ by

$$
\left\{\begin{array} { l } 
{ V _ { 1 } = \alpha - 1 } \\
{ V _ { 2 } = \frac { 1 } { n ^ { 2 } } ( \alpha ^ { 2 } - \alpha ( 6 + n ^ { 2 } ) + 4 + n ^ { 2 } ) } \\
{ V _ { 3 } = 1 }
\end{array} \text { and } \left\{\begin{array}{l}
H_{1}=2 V_{1} \\
H_{2}=n\left(V_{1}+V_{2}\right) \\
H_{3}=V_{1}+V_{3}
\end{array}\right.\right.
$$

then $V$ and $H$ are positive, namely $V_{i}>0$ and $H_{i}>0$ for $i=1,2,3$.

Proof. If $A$ is the symmetric matrix defined by $A=\left(\begin{array}{ccc}5+n^{2} & n^{2} & 1 \\ n^{2} & n^{2} & 0 \\ 1 & 0 & 1\end{array}\right)$, then the characteristic polynomial $\chi_{A}$ is $P$. This ensures that $P$ has only reals roots. In addition $A$ is an irreducible matrix, indeed $\left(A^{2}\right)_{i j}>0$ for all $i, j$. This proves that $\alpha$ is the PerronFrobenius eigenvalue of the entire matrix $A$ and therefore $\alpha>1$. Let us show that $V$ is an eigenvector for the eigenvalue value $\alpha$ of the matrix $A$. The Perron-Frobenius theorem will then show the positivity of $V$.

For that we have to show that $V$ is a solution of the linear system

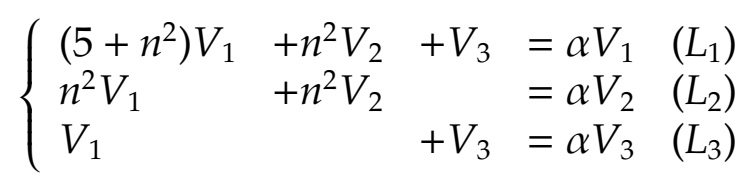

This is a simple verification, according to the fact that $\alpha^{3}=2\left(n^{2}+3\right) \alpha^{2}-\left(7 n^{2}+\right.$ 4) $\alpha+4 n^{2}$. The positivity of $H$ is then clear. 
Thanks to the previous statement, for any $n \geq 1$, let $\left(X_{n}, \omega_{n}\right)$ be the surface presented in Figure 10.

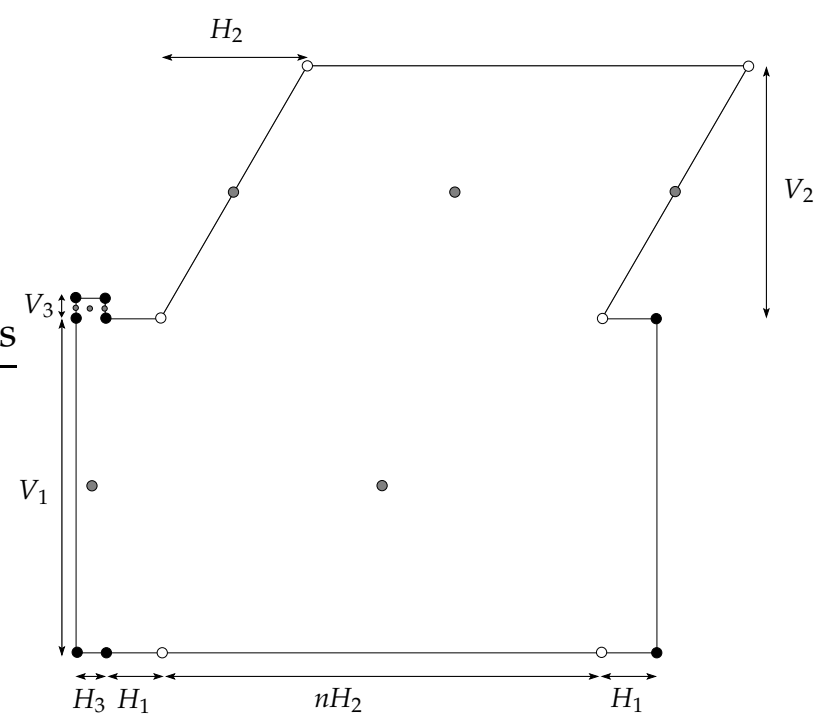

Figure 10. A surface $\left(X_{n}, \omega_{n}\right)$ in the hyperelliptic locus $\mathcal{L}$. The vertical direction has been stretched.

Lemma 6.3. The surface $\left(X_{n}, \omega_{n}\right)$ is a bouillabaisse surface. Moreover its trace field is $\mathbb{Q}\left(\alpha_{n}\right)$ and its trace field has degree 3 over $\mathbb{Q}$ if $n>2$.

Proof. According to the horizontal direction, the surface is decomposed into a completely periodic 3C-direction (see Figure 10). Let us calculate the moduli of theses cylinders and show that they are equal. Using $\left(L_{1}\right)$ and $\left(L_{2}\right)$ in the system (3), one checks that the moduli are

$$
\frac{H_{3}}{V_{3}}=V_{1}+V_{3}=\alpha, \quad \frac{H_{3}+2 H_{1}+n H_{2}}{V_{1}}=\alpha, \quad \text { and } \quad \frac{n H_{2}}{V_{2}}=n^{2} \frac{V_{1}+V_{2}}{V_{2}}=\alpha .
$$

All the moduli are equal, thus $\left(X_{n}, \omega_{n}\right)$ is stabilized by the parabolic element $T_{\alpha}=\left(\begin{array}{ll}1 & \alpha \\ 0 & 1\end{array}\right)$.

Now let us consider the vertical direction; this is a completely periodic $2 T_{\text {fix }} 2 \mathrm{C}$ direction. The two simple cylinders have moduli $V_{1} / H_{1}=1 / 2$. The two others cylinders have moduli $\left(V_{1}+V_{3}\right) / H_{3}=1$ and $n\left(V_{1}+V_{2}\right)\left(H_{2}\right)=1$. Thus the parabolic element $U_{1 / 2}=\left(\begin{array}{cc}1 & 0 \\ \frac{1}{2} & 1\end{array}\right)$ stabilises $\left(X_{n}, \omega_{n}\right)$.

Therefore $\left(X_{n}, \omega_{n}\right)$ is a bouillabaisse surface, since it has two transverse parabolic directions. Moreover $\left(X_{n}, \omega_{n}\right)$ is stabilised by the hyperbolic element $T U$ which has the trace $\frac{1}{2}(4+\alpha)$. The trace field of $\left(X_{n}, \omega_{n}\right)$ is then $\mathbb{Q}\left(\alpha_{n}\right)$. The next lemma shows that $P_{n}$ is irreducible over $\mathbb{Q}$ if $n \geq 3$ which will end the proof. 
Lemma 6.4. Let $n$ be a positive integer. The polynomial $P_{n}(X)=X^{3}-2\left(n^{2}+3\right) X^{2}+$ $\left(7 n^{2}+4\right) X-4 n^{2}$ is irreducible over $\mathbb{Q}$ if $n>2$.

Proof. Since $P_{n}$ is monic, it is irreducible over $\mathbb{Q}$ if and only if it is irreducible over $\mathbb{Z}$. As it is of degree 3 , it is irreducible over $\mathbb{Z}$ if and only if it has no root in $\mathbb{Z}$. We check the following equalities:

$$
\left\{\begin{array}{ll}
P_{n}(0) & =-4 n^{2} \\
P_{n}(1) & =-1+n^{2} \\
P_{n}(2) & =-8+2 n^{2} \\
P_{n}(3) & =-n^{2}-15 \\
P_{n}\left(2 n^{2}+2\right) & =-8-14 n^{2}-2 n^{4} \\
P_{n}\left(2 n^{2}+3\right) & =-15-11 n^{2}+2 n^{4},
\end{array} \quad \text { thus for } n \geq 2 \quad \begin{cases}P_{n}(0) & <0 \\
P_{n}(1) & >0 \\
P_{n}(2) & >0 \\
P_{n}(3) & <0 \\
P_{n}\left(2 n^{2}+2\right)<0 \\
P_{n}\left(2 n^{2}+3\right)>0\end{cases}\right.
$$

By the intermediate value theorem, if $n>2, P_{n}$ has 3 real roots $\alpha, \beta, \gamma$ satisfying:

$$
\left\{\begin{array}{l}
0<\gamma<1 \\
2<\beta<3 \\
2 n^{2}+2<\alpha<2 n^{2}+3
\end{array}\right.
$$

Consequently, $P_{n}$ has no root in $\mathbb{Z}$ thus it is irreducible over $\mathbb{Z}$.

Remark 6.4.1. The polynomials $P_{1}$ and $P_{2}$ are not irreducible; indeed, if $n=1,2$ we have $P_{1}=(X-1)\left(X^{2}-7 X+4\right)$ and $P_{2}=(X-2)\left(X^{2}-12 X+8\right)$. More precisely, $\left(X_{1}, \omega_{1}\right)$ is an unramified cover of a Veech surface belonging to $\mathcal{H}(2)$.

\subsection{Another completely periodic (but non-parabolic) direction on $\left(X_{n}, \omega_{n}\right)$.}

Proposition 6.5. Let $\Theta$ be the slope $V_{2} / H_{2}=n / \alpha$. Then the direction $\Theta$ on $\left(X_{n}, \omega_{n}\right)$ is completely periodic $2 T_{\mathrm{fix}} 2 C$-direction on $\left(X_{n}, \omega_{n}\right)$. Moreover, if $n>1$, this direction is not parabolic.

We decompose the proof into three steps. We first prove that the direction $\Theta$ is completely periodic. We then compute the moduli of the cylinders and finally we prove that one ratio is not rational.

Lemma 6.6. The direction $\Theta$ is a completely periodic $2 T_{\text {fix }} 2 C$-direction.

Proof. By definition, $\gamma_{5}$ is closed and the corresponding holonomy vector is

$$
\int_{\gamma_{5}} \omega_{n}=\left(\begin{array}{c}
H_{2} \\
V_{2}
\end{array}\right) .
$$

Let us prove that $\gamma_{4}$ is also closed. Let $x_{i}$ be the $x$-coordinates of intersection point of $\gamma_{4}$ with the top (and bottom) of the horizontal cylinder of height $V_{1}$ as 


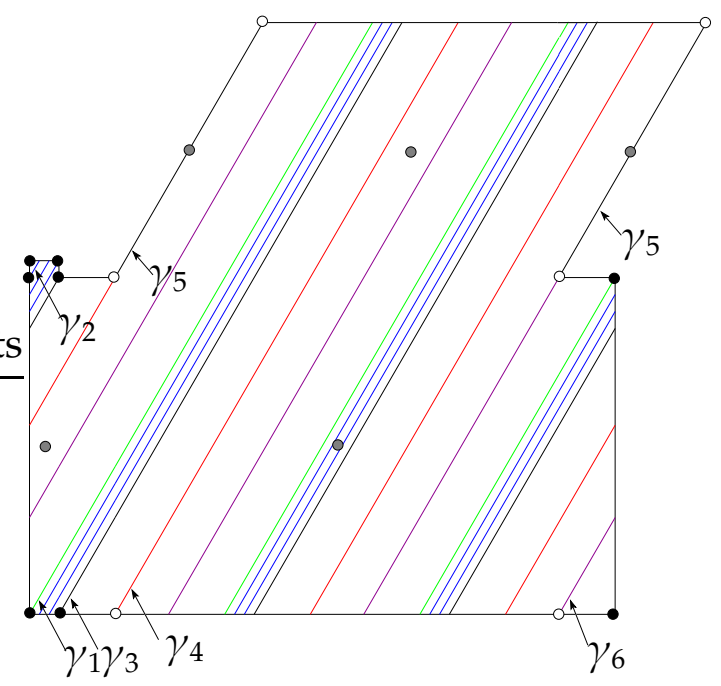

Figure 11. Another completely periodic (non parabolic) $2 T_{\text {fix }} 2 C$ direction on $\left(X_{n}, \omega_{n}\right)$.

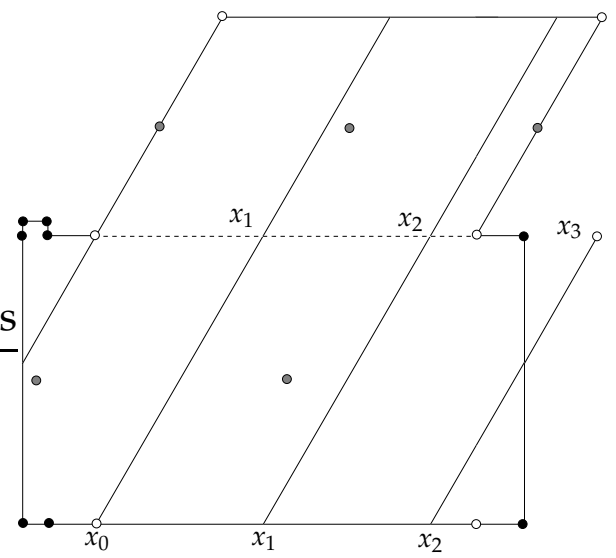

Figure 12. A periodic direction on $\left(X_{3}, \omega_{3}\right)$ in the direction $\Theta$.

illustrated in Figure 12. We will show that $x_{i}<H_{1}+H_{3}+n H_{2}$ for all $i=1, \ldots, n-1$ and $x_{n}=3 \mathrm{H}_{1}+2 \mathrm{H}_{3}+n \mathrm{H}_{2}$ which will ensure that $\gamma_{4}$ is closed.

Elementary geometry gives $\frac{V_{1}}{x_{i}-x_{i-1}}=\Theta=\frac{n}{\alpha}$, which yields

$$
x_{i}=\frac{\alpha V_{1}}{n}+x_{i-1}=\frac{i \cdot \alpha V_{1}}{n}+x_{0}=\frac{i \cdot \alpha V_{1}}{n}+H_{1}+H_{3} .
$$


Let $\Delta_{i}=x_{i}-\left(H_{1}+H_{3}+n H_{2}\right)$. Recall that $n H_{2}=\alpha V_{2}$ and $V_{1}=\left(\frac{\alpha}{n^{2}}-1\right) V_{2}$ (thanks to $\left(L_{2}\right)$ in Equation (3) $)$. Then

$$
\Delta_{i}=\frac{i \cdot \alpha V_{1}}{n}-\alpha V_{2}=\frac{\alpha V_{2}}{n}\left(i\left(\frac{\alpha}{n^{2}}-1\right)-n\right) .
$$

Claim 6.7. One has

$$
\frac{\alpha}{n^{2}}-1<\frac{n}{n-1}
$$

Proof of the Claim. If $n>2$, we already proved that $\alpha<2 n^{2}+3$ (see Equation (4)). Hence the claim follows, once we have shown $\frac{2 n^{2}+3}{n^{2}}-1=1+\frac{3}{n^{2}}<\frac{n}{n-1}$. This is obvious if $n>2$. The case $n=2$ is checked directly.

Therefore, if $i \leq n-1$ we have

$$
\Delta_{i}<\frac{\alpha V_{2}}{n}\left((n-1) \cdot \frac{n}{n-1}-n\right)=0 .
$$

To complete the proof, one has to show that $x_{n}=3 \mathrm{H}_{1}+2 \mathrm{H}_{3}+n \mathrm{H}_{2}$. Equation (5) with $i=n$ gives $x_{n}=\alpha V_{1}+H_{1}+H_{3}$. Therefore

$$
\begin{aligned}
x_{n}-\left(3 H_{1}+2 H_{3}+n H_{2}\right)=\alpha V_{1}- & 4 V_{1}-V_{1}-V_{3}-n^{2}\left(V_{1}+V_{2}\right)= \\
= & \left(5+n^{2}\right) V_{1}+n^{2} V_{2}+V_{3}-\alpha V_{1}=0 \quad \text { by }\left(L_{1}\right) .
\end{aligned}
$$

Hence $x_{n}=3 H_{1}+2 H_{3}+n H_{2}$ and $\gamma_{4}$ is closed. In addition, we get the holonomy vector associated to $\gamma_{4}$ :

$$
\int_{\gamma_{4}} \omega_{n}=\left(\begin{array}{c}
* \\
(n-1)\left(V_{1}+V_{2}\right)+V_{1}
\end{array}\right) .
$$

The same calculation shows that $\gamma_{1}, \gamma_{3}$ and $\gamma_{6}$ are also closed. It is not difficult to check what is the complement of these four saddle connections: it gives two tori. Hence the surface $\left(X_{n}, \omega_{n}\right)$ is decomposed into a $2 T_{f i x} 2 C$-direction in the direction $\Theta$ (see Figure 13). The torus $T_{1}$ is actually periodic. By Theorem 3.8 the SAF-invariant vanishes in this direction and the SAF-invariant is equals to zero on cylinders $C$ and torus $T_{1}$. Therefore the SAF-invariant is also equal to zero on $T_{2}$ and finally $\Theta$ is completely periodic. The lemma is proven. 


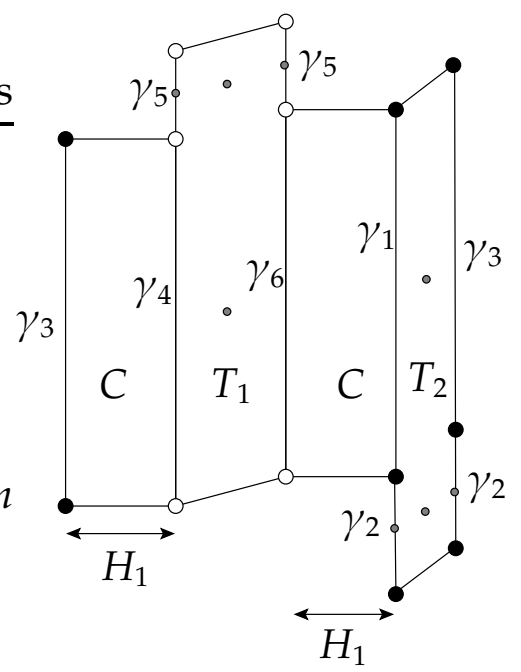

FIgURE 13. The decomposition of $\left(X_{n}, \omega_{n}\right)$ in the direction $\Theta$ : this is a completely periodic $2 T_{\text {fix }} 2 C$-direction.

Lemma 6.8. If $C, T_{1}, T_{2}$ denote the cylinder decomposition in the direction $\Theta$, then the ratio of the moduli of $C$ and $T_{1}$ is

$$
r_{1}=\frac{m(C)}{m\left(T_{1}\right)}=\frac{(\alpha-n)\left(2 n^{3}-n^{2}-(n-1) \alpha\right)}{2 n\left(\alpha-n^{2}\right)} .
$$

Proof. According to Figure 13, we can compute the circumference and the height of the cylinders $C$ and $T_{1}$. The circumference of $C$ is just $\left|\gamma_{4}\right|$ where

$$
\left|\gamma_{4}\right|^{2}=\left((n-1)\left(V_{1}+V_{2}\right)+V_{1}\right)^{2}\left(1+\frac{1}{\Theta^{2}}\right)
$$

and the height is $H_{1}$.

The circumference of $T_{1}$ is just $\left|\gamma_{4}\right|+\left|\gamma_{5}\right|$ where

$$
\left|\gamma_{5}\right|^{2}=V_{2}^{2}\left(1+\frac{1}{\Theta^{2}}\right)
$$

and the height is $h\left(T_{1}\right)=\left(H_{1}+H_{3}+n H_{2}\right)-x_{n-1}$ (see Figure11). This can be simplified to

$$
h\left(T_{1}\right)=n H_{2}-\frac{(n-1) \alpha V_{1}}{n}=\alpha V_{2}-\frac{(n-1) \alpha}{n}\left(\frac{\alpha}{n^{2}}-1\right) V_{2}=\frac{\alpha V_{2}}{n^{3}}\left(2 n^{3}-n^{2}-(n-1) \alpha\right) .
$$


Taking the ratio we get:

$$
r_{1}=\frac{\frac{\left|\gamma_{4}\right|}{H_{1}}}{\frac{\left|\gamma_{4}\right|+\left|\gamma_{5}\right|}{h\left(T_{1}\right)}}=\frac{\left|\gamma_{4}\right|}{\left|\gamma_{4}\right|+\left|\gamma_{5}\right|} \cdot \frac{h\left(T_{1}\right)}{H_{1}} .
$$

The quantities on the right hand side can be simplified to

$$
\frac{\left|\gamma_{4}\right|}{\left|\gamma_{4}\right|+\left|\gamma_{5}\right|}=\frac{(n-1)\left(V_{1}+V_{2}\right)+V_{1}}{(n-1)\left(V_{1}+V_{2}\right)+V_{1}+V_{2}}=\frac{\frac{1}{n} \alpha V_{2}-V_{2}}{\frac{1}{n} \alpha V_{2}}=\frac{\alpha-n}{\alpha}
$$

and

$$
\frac{h\left(T_{1}\right)}{H_{1}}=\frac{\frac{1}{n^{3}} \alpha V_{2}\left(2 n^{3}-n^{2}-(n-1) \alpha\right)}{2 V_{1}}=\frac{\alpha\left(2 n^{3}-n^{2}-(n-1) \alpha\right)}{2 n\left(\alpha-n^{2}\right)} .
$$

Plugging this into the previous equation, this gives

$$
r_{1}=\frac{(\alpha-n)\left(2 n^{3}-n^{2}-(n-1) \alpha\right)}{2 n\left(\alpha-n^{2}\right)}
$$

which is the desired equality.

Lemma 6.9. The following are equivalent:

(1) $r_{1} \in \mathbb{Q}$.

(2) $r_{1}=\frac{1}{2}$.

(3) $n=1$.

Proof. If $r_{1} \in \mathbb{Q}$, using equation (6), we get in the basis $\left\{1, \alpha, \alpha^{2}\right\}$ :

$$
\begin{cases}n^{3}\left(2 n-1-2 r_{1}\right) & =0 \\ n\left(2 n^{2}-1-2 r_{1}\right) & =0 \\ n-1 & =0\end{cases}
$$

The solution gives $r_{1}=\frac{1}{2}$ and $n=1$. The lemma is proven.

7. Surfaces With a $2 T_{\text {fix }} 2$-Direction and covering constructions

The locus $\mathcal{D} \subset \mathcal{L}$ of translation surfaces having a completely periodic direction of type $2 T_{\text {fix }} 2 C$ has proven to be useful above. Contrary to genus two, where pairs of saddle connections exchanged by the hyperelliptic involution always exist ([Mc3] $), \mathcal{D}$ is not equal to $\mathcal{L}$.

In order to show this, we consider coverings $\pi: X \rightarrow Y$ of a flat surface $(Y, \eta)$ in $\mathcal{H}(2)$. Such a covering is unramified and has degree two. Given $Y$, the covering $\pi$ is determined by the choice of $\zeta \in H^{1}(Y, \mathbb{Z} / 2)$ or of a line bundle $\mathcal{M}$ with $\mathcal{M}^{2}=O_{Y}$. 
Recall (e.g. [KZ03] ) that on $Y$ we have a natural spin structure (i.e. a square root of the canonical bundle) given by $O_{Y}(P)$, if $Z(\eta)=2 P$. Since $P$ is a Weierstrass point, this spin structure has odd parity. $O_{Y}(P) \otimes \mathcal{M}$ defines another spin structure on $Y$. By [Ati71], the space $C$ of coverings $\pi$ has two components, distinguished by the parity of this spin structure $h^{0}\left(Y, O_{Y}(P) \otimes \mathcal{M}\right) \bmod 2$. Let $Z(\omega)=2 P_{1}+2 P_{2}$ be the preimage of $Z(\eta)$. Since

$$
H^{0}\left(X, \mathcal{O}_{X}\left(P_{1}+P_{2}\right)\right) \cong H^{0}\left(Y, \mathcal{O}_{Y}(P) \otimes \mathcal{M}\right)+H^{0}\left(Y, \mathcal{O}_{Y}(P)\right)
$$

this parity is given by the usual parity of the spin structure on $X$. Consequently, precisely one of the components of $C$ lies in $\Omega M_{3}(2,2)^{\text {odd }}$ and in fact automatically in the hyperelliptic locus $\mathcal{L}$. We denote this component by $C^{\text {odd }}$.

Theorem 7.1. The locus $\mathcal{D}$ is strictly contained in $\mathcal{L}$. More precisely, the intersection $\mathcal{D}^{c} \cap C^{\text {odd }}$ is non-empty and strictly contained in $C^{\text {odd }}$. It consists of orbits of Veech surfaces.

Proof. First we show that the intersection is not empty. Consider a square-tiled covering of 6 squares as Figure 14 .

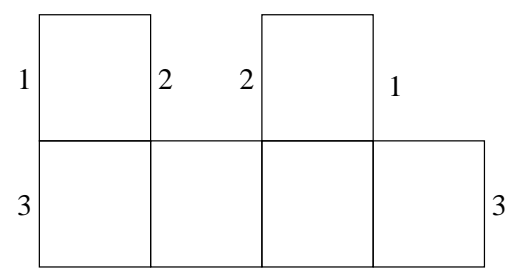

FIgURE 14. A square-tiled surface in $\mathcal{L}$ without $2 T_{\text {fix }} 2 C$-direction

Horizontal sides are glued by vertical translations. Using e.g. [Sch04], one checks that the affine group of this square-tiled covering has three cusps, given by the horizontal, and vertical direction and by the direction of slope 1 . None of these directions is a $2 T_{\text {fix }} 2 C$-direction.

By [Cal04], a surface in $\mathcal{H}(2)$ which is not Veech has a direction where it splits into a cylinder and a non-periodic torus. Consequently, a covering of a generic surface in $\mathcal{H}(2)$ yields a surface in $\mathcal{L}$ with an irrational $2 T 2 C_{\text {fix }}$-direction. Such a surface also has a $2 T_{\text {fix }} 2 C$-direction by Figure 6 (b).

Since the saddle connections involved in a $2 T_{\text {fix }} 2 C$-direction are homologous, $\mathcal{D}$ is open. Consequently, $\mathcal{D}^{c} \cap C^{\text {odd }}$ is closed and $\mathrm{SL}_{2}(\mathbb{R})$-invariant. The last claim now follows from [Mc3]. 


\section{REFERENCES}

[Arn81] P. Arnoux - "Un invariant pour les échanges d'intervalles et les flots sur les surfaces (French)", Thèse, Université de Reims (1981).

[Arn88] _ _ "Un exemple de semi-conjugaison entre un échange d'intervalles et une translation sur le tore (French)", Bull. Soc. Math. France 116 (1988), no. 4, pp. 489-500

[AY81] P. Arnoux and J.C. Yoccoz - "Construction de difféomorphismes pseudo-Anosov (French)", C. R. Acad. Sci. Paris Sér. I Math. 292 (1981), no. 1, pp. 75-78.

[Ati71] M. Aттуaн - "Riemann surfaces and spin structures", Ann. Sci. Éc. Norm. Sup. 4 (1971), pp. 47-62.

[Cal04] K. Calta - "Veech surfaces and complete periodicity in genus two", J. Amer. Math. Soc. 17 (2004), no. 4, pp. 871-908.

[CS07] K. Calta and J. Smillie - "Algebraically periodic translation surfaces", preprint, math.DS/0703567(2007).

[EMS03] A. Eskin, H. Masur and M. Schmoll - "Billiards in rectagles with barriers ", Duke Math. J. 118 (2003), no. 3, pp. 427-463

[FLP] A. Fathi, F. Laudenbach and V. Pó́naru - "Travaux de Thurston sur les surfaces", Astérisque 66-67 (1979).

[HLe06] P. Hubert and S. Lelievre - "Prime arithmetic Teichmüller discs in $\mathcal{H}(2)$ ", Isreal J. Math 151 (2006), pp. 281-321

[HLa06] P. Hubert and E. Lanneau - "Veech groups with no parabolic element", Duke Math. J. 133 (2006), no. 2, pp. 335-346.

[HLM06] P. Hubert, E. Lanneau and M. Möller, "The Arnoux-Yoccoz Teichmüller disc", preprint, math.GT/0611655(2006).

[KSO0] R. KenYon and J. Smillie - "Billiards in rational-angled triangles", Comment. Math. Helv. 75 (2000), pp. 65-108.

[KZ03] M. KontSEvich and A. Zorich - "Connected components of the moduli spaces of Abelian differentials with prescribed singularities", Invent. Math. 153 (2003), no. 3, pp. 631-678.

[Lan04] E. LANNEAU - "Hyperelliptic components of the moduli spaces of quadratic differentials with prescribed singularities", Comm. Math. Helv. 76 (2004), pp. 471-501.

[MT02] H. Masur and S. TAвасHNikov - "Rational billiards and flat structures", Handbook of dynamical systems Vol. 1A, (2002), North-Holland, Amsterdam, pp. 1015-1089.

[Mas82] H. MAsur - "Interval exchange transformations and measured foliations", Ann. of Math. (2) 115 (1982), no. 1, pp. 169-200.

[Mas86] __ "Closed trajectories for quadratic differentials with an application to billiards", Duke Math. J. 53 (1986), no. 2, pp. 307-314.

[Mc1] C. McMullen - "Billiards and Teichmüller curves on Hilbert modular surfaces", J. Amer. Math. Soc. 16 (2003), no. 4, pp. 857-885.

[Mc2] _ "Teichmüller geodesics of infinite complexity", Acta Math. 191 (2003), no. 2, pp. 191-223.

[Mc3] , "Dynamics of $\mathrm{SL}_{2}(\mathbb{R})$ over moduli space in genus two", Ann. of Math. to appear.

[Mc4] _ "Teichmüller curves in genus two: Discriminant and spin", Math. Ann. 333 (2005), pp. 87-130.

[Mc5] , "Teichmüller curves in genus two: The decagon and beyond", J. reine angew. Math. 582 (2005), pp. 173-200. 
[Mc6] , "Teichmüller curves in genus two: Torsion divisors and ratios of sines", Invent. Math. 165 (2006), pp. 651-672.

[Rat91] M. RatNeR - "Raghunathan's topological conjecture and distributions of unipotent flows", Duke Math. J. 63 (1991), no. 1, pp. 235-280.

[Sch04] G. Sснміттнüsen - "An algorithm for finding the Veech group of an origami", Exp. Math. 13 (2004), pp. 459-472.

[Thu88] W. Thurston - "On the geometry and dynamics of diffeomorphisms of surfaces", Bull. A.M.S. 19 (1988), pp. 417-431.

[Vee82] W. VEECH - "Gauss measures for transformations on the space of interval exchange maps", Ann. of Math. (2) 115 (1982), no. 1, pp. 201-242.

[Vee86] _ _ "The Teichmüller geodesic flow", Ann. of Math. 124 (1986), p. 441-530.

[Vee89] _ "Teichmüller curves in the moduli space, Eisenstein series and an application to triangular billiards", Invent. Math. 97 (1989), p. 533-683.

[Vee90] _ _Moduli spaces of quadratic differentials", J. Analyse Math. 55 (1990), p. 117-170.

Laboratoire d'Analyse, Topologie et Probabilités (LATP)

Case cour a Faculté de Saint Jérôme Avenue Escadrille Normandie-Niemen

13397, Marseille cedex 20, France

E-mail address: hubert@cmi .univ-mrs.fr

Centre de Physique Théorique (CPT), UMR CNRS 6207

Université du Sud Toulon-VAR AND

Fédération de Recherches des Unités de Mathématiques de Marseille

Luminy, Case 907, F-13288 Marseille Cedex 9, France

E-mail address: lanneau@cpt.univ-mrs.fr

Max-Planck-Institut für Mathematik

Postfach 7280

53072 BonN, GERMANY

E-mail address: moeller@mpim-bonn.mpg.de 